\title{
Cuticular Wax Composition is Essential for Plant Recovery Following Drought with Little Effect under Optimal Conditions
}

Boaz Negin, Shelly Hen-Avivi, Efrat Almekias-Siegl, Lior Shachar and Asaph Aharoni Department of Plant and Environmental Sciences, Weizmann Institute of Science, Rehovot, Israel

\begin{abstract}
Despite decades of extensive study, the role of cuticular lipids in sustaining plant fitness is far from being understood. To answer this fundamental question, we employed genome editing in tree tobacco (Nicotiana glauca) plants and generated mutations in 16 different cuticular lipids-related genes. We chose tree tobacco due to the abundant, yet simply composed epicuticular waxes deposited on its surface. Five out of 9 different mutants that displayed a cuticular lipids-related phenotype were selected for in depth analysis. They had either reduced total wax load or complete deficiency in certain wax components. This led to substantial modification in surface wax crystal structure and to elevated cuticular water loss. Remarkably, under non-stressed conditions, mutant plants with altered wax composition did not display elevated transpiration or reduced growth. However, once exposed to drought, plants lacking alkanes were not able to strongly reduce their transpiration, leading to leaf death and impaired recovery upon resuscitation, and even to stem cracking, a phenomenon typically found in trees experiencing drought stress. In contrast, plants deficient in fatty alcohols exhibited an opposite response, having reduced cuticular water loss and rapid recovery following drought. This differential response was part of a larger trend, of no common phenotype connecting plants with a glossy appearance. We conclude that alkanes are essential under drought response and much less under normal non-stressed conditions, enabling plants to seal their cuticle upon stomatal closure, reducing leaf death and facilitating a speedy recovery.
\end{abstract}




\section{Introduction}

Plants aerial organs are exposed to a plethora of conditions that require a barrier to shield their inner tissues. Such layers protect them from many factors including a desiccating environment, high radiation in different wavelengths and colonization or feeding by other organisms such as fungi, bacteria, insects and even grazing mammals. To cope with these, plants developed a complex surface layer, which at times may also contain specialized components that aid the plant in its response to the different factors. The cuticle may be imbedded with flavonoids (Ryan et al., 2001; Ryan et al., 2002) and anthocyanins (ChalkerScott, 1999) reducing UVB damage to the mesophyll. Trichomes present on the surface may serve as a structural and chemical obstacle for plant herbivores (Hanley et al., 2007) and change leaf reflectance, reducing photoinhibition and UV-B related damage (Steffens and Walters, 1991; Peiffer et al., 2009; Sonawane et al., 2020; Bickford, 2016). The plant cuticle consists of three core elements localized beyond the epidermis cells. The cutin polymer composed of an amorphous matrix largely comprising $\mathrm{C}_{16}$ and $\mathrm{C}_{18}$ fatty acids connected in esteric bonds (Yeats and Rose, 2013), intracuticular wax embedded in the cutin layer and epicuticular waxes secreted beyond the cutin. Though it is clear that the cuticle plays a crucial role in preventing non stomatal water loss, the extent to which epicuticular wax contributes to this varies widely and in some cases may be negligible compared to the other two elements (Jetter and Riederer, 2016; Zeisler and Schreiber, 2016; Zeisler-Diehl et al., 2018). This keeps open the question of epicuticular wax's function, in cases where its contribution to the transpirational barrier in small. A related question, is to what extent the different wax components contribute to the different functions of epicuticular waxes.

Decades of extensive research, mainly in the model species Arabidopsis thaliana resulted in elucidation of most biosynthetic pathways associated with production of the different wax components (Fig. 1; Lee and Suh, 2015). In Arabidopsis, fatty acids synthesized in plastids are transported to the endoplasmic reticulum (ER) where they are converted to acyl CoA by LONG CHAIN ACYL SYNTHETASE (LACS; Schnurr et al., 2004; Lü et al., 2009). The fatty acid elongase complex composed of four proteins then elongates these $\mathrm{C}_{16}-\mathrm{C}_{18}$ precursors. These include (i) $\beta$-ketoacyl-CoA synthase (KCS; Millar and Kunst, 1997) having different variants responsible for elongation of certain acyl substrates (e.g. KCS6 targeted here is responsible for elongation from 24 to 34 carbons (Millar et al., 1999), (ii) $\beta$ ketoacyl-CoA reductase (KCR; Beaudoin et al., 2009), (iii) 3-hydroxyacyl-CoA dehydratase 
(HCD; Bach et al., 2008) and (iv) trans-2,3-enoyl-CoA reductase (ECR; Zheng et al., 2005).

These

enzymes

elongate

the

Acyl

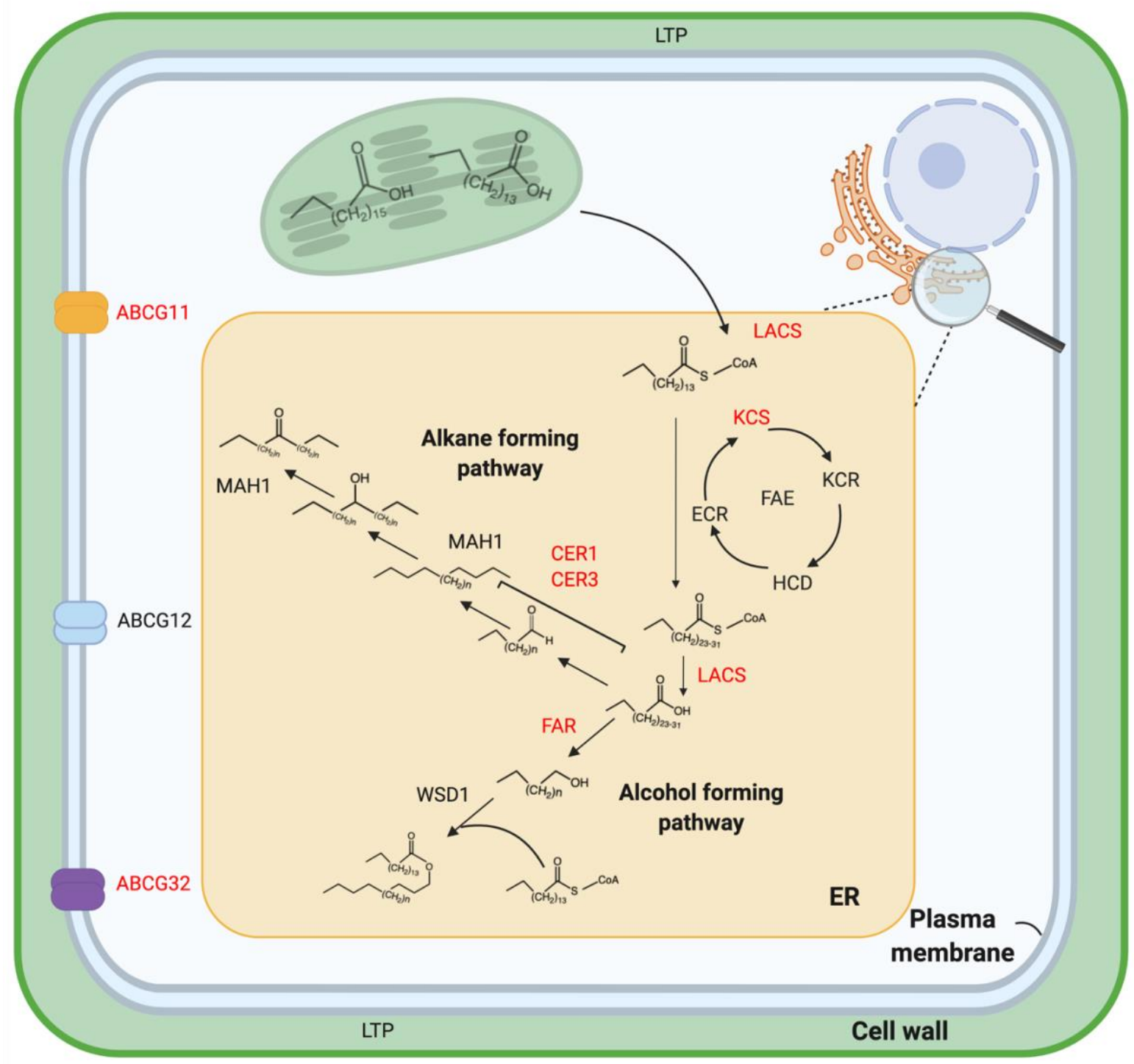

Figure 1. A schematic model of epicuticular wax biosynthesis and transport pathways.

Not all proteins involved in these pathways are presented and genes corresponding to proteins marked in red were targeted for editing and exhibited a visual phenotype in this study. The scheme presents fatty acid elongation and division to the two main wax synthesis pathways the alkane and fatty alcohol forming pathways. Genes silenced that are involved in wax synthesis but not a specific pathway include KCS6 and LACS. Those taking part in alkane synthesis are CERI,CER3 and MAH1, and fatty alcohol synthetic genes include FAR and WSD1.

CoAs through a cycle in which malonyl CoA and the acyl are condensed and at its end the acyl is elongated by two carbons. The outcome of the elongation process are very long chain 
fatty (VLCF) acyl CoA of different lengths used in several epicuticular wax biosynthesis pathways. They can be converted into very long chain fatty acids (VLCFA) or enter two paths, the alkane- and alcohol- forming pathways. In the alkane-forming pathway, VLCF acyl-CoAs are decarboxylated to create aldehydes and then alkanes. The exact enzymatic mechanisms taking place during the conversion of acyl-CoA to alkanes are not clear, though it is known that ECERIFERUM1 (CER1) and ECERIFERUM 3 (CER3) take part in the first stages of conversion to alkanes (Aarts et al., 1995; Chen et al., 2003; Bourdenx et al., 2011). Following the synthesis of alkanes, these can be further modified to form secondary alcohols and ketones, by the MID-CHAIN ALKANE HYDROXYLASE1 (MAH1); a cytochrome p450 catalyzing both reactions (Greer et al., 2007). In the primary alcohol forming pathway, VLCF acyl-CoAs are converted to primary alcohols through the addition of a hydroxyl at the acyls end. This step is catalyzed by the FATTY ACYL-COA REDUCTASE (FAR) enzyme (Rowland et al., 2006). An additional stage in the alcohol forming pathway is the conjunction of $\mathrm{C}_{16}$ or $\mathrm{C}_{18}$ fatty acids to the primary alcohols to create wax esters by the bifunctional wax synthase/ acyl CoA:diacylglycerol acyltransferase1 (WSD1; Li et al., 2008). Although the presence of epicuticular wax is almost ubiquitous among plants, there is great diversity between species and even different organs of the same plant, in chemical structure and form of wax crystals they produce (Barthlott et al., 1998; Lee and Suh, 2015).

Tree tobacco (Nicotiana glauca) is a perineal shrub originating in South America which has since spread worldwide. The appearance of its stems and leaves is glaucous, an uncommon appearance in tobacco species which gave the species its name. This glaucous appearance, is the result of a high load of epicuticular wax coating the plant's aerial organs. This wax is composed almost solely of $\mathrm{C}_{31}$ alkanes with lesser amounts of additional alkanes, fatty alcohols and aldehydes (Mortimer et al., 2012). Furthermore, N. glauca accumulates substantial amounts of wax in response to drought, while maintaining its original composition (Cameron et al., 2006). These characteristics including the availability of its genome sequence (Usadel et al., 2018), efficient stable transformation and the ease of dissecting its epidermis layer make it an excellent model plant for studying the yet unresolved role of epicuticular waxes in the plant life cycle.

In this study, we employed CRISPR-Cas9 based technology to edit 16 cuticular lipids metabolism genes and generate mutants in N. glauca. Following initial characterization, we carried out in-depth research on five selected knockout mutants that showed diverse patterns of wax composition. This mutant set was subjected to a range of examinations to help us better understand what the contribution of epicuticular wax is to plant fitness, and to attribute 
this contribution to specific wax components. We found, that under optimal conditions epicuticular wax has little effect on plant fitness. In contrast, following drought the alkane fraction is essential for plant recovery, whereas deficiency in fatty alcohols did not affect plants negatively under our experimental conditions. Findings in this study highlight the specific role of plant epicuticular wax in episodes of drought and the following recovery phase. They further explain how diversity of wax components and structures contribute to plant fitness under abiotic stress conditions.

\section{Results}

Transcriptomics in Nicotiana glauca epidermis tissues under drought conditions facilitates the discovery of cuticular lipids-related genes

The most striking feature of $N$. glauca is the glaucous waxy appearance formed by an extremely high but very simply composed wax layer covering its above ground organs. Such intense wax coverage provokes a fundamental question with respect to the high carbon investment in epicuticular wax production set against its contribution to plant fitness. Which wax component contributes to fitness and in what manner is a consequent key question. To answer these, it was first essential to identify cuticular lipids-associated genes active in the $N$. glauca epidermis layer. We thus performed transcriptomic analysis of five different shoot tissues including dissected adaxial and abaxial leaf epidermis, stem epidermis, complete leaves, and whole stems. Expression profiling was carried out in well-watered plants or those under drought conditions, as a previous study demonstrated drought-induced wax formation in N. glauca leaves (Cameron et al., 2006). To detect genes associated with cuticular lipids formation we next mined the transcriptome dataset for transcripts that were epidermis enriched, drought induced or a combination of the two conditions. We found that many homologs of cuticular lipids metabolism genes (e.g. KCS6, CER1, FAR and ABCG32), displayed a similar 'expression signature'; high epidermal enrichment and mild drought induction (Fig. 2). Out of tens of genes putatively associated with cuticular lipids metabolism we selected 16 for further study. Editing these genes could alter wax and cutin synthesis and transport in a variety of manners resulting in a set of mutant lines with diverse was compositions (Table S1). This set comprised seven genes putatively encoding wax 
biosynthesis enzymes including LACS1, KCS6, CER1, CER3, MAH1, FAR and WSD1 and three cutin biosynthesis enzymes - Glycerol-3-phosphate 2-O-acyltransferase 4 (GPAT4), Cytochrome P450 86A22 (CYP86A22) and GDSL-motif esterase/acyltransferase/lipase $(G D S L)$. Three putative transporters - ATP-BINDING CASSETTE G11 (ABCG11), ATPBINDING CASSETTE G32 (ABCG32) and ACYL-COA-BINDING PROTEIN 1 (ACBP1) and three transcription factor homologs - SHINE1, SHINE3 and MYB96, complemented the set of selected genes. We next generated plant transformation vectors for editing the 16-gene set through CRISPR-Cas9 technology and introduced them to N. Glauca (Fig. S2-S10).

A

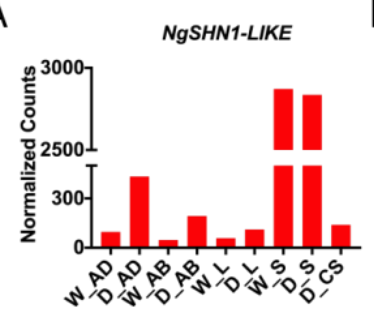

$\mathrm{E}$

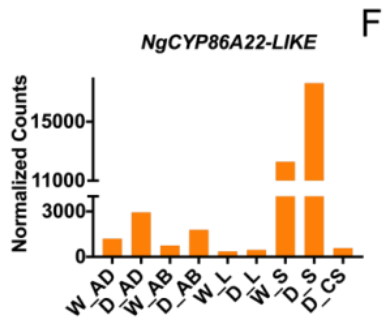

I

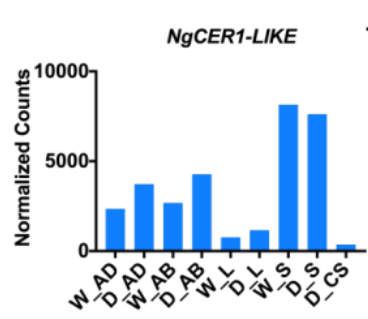

M

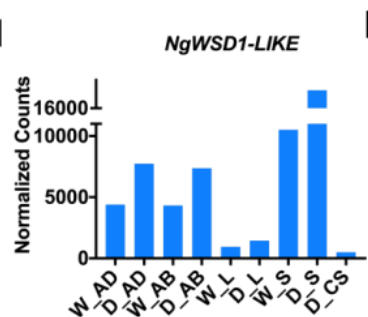

B

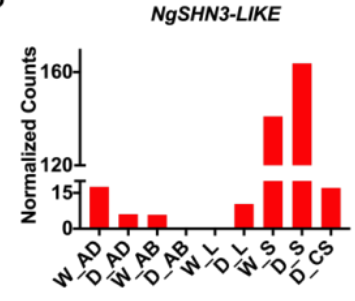

F

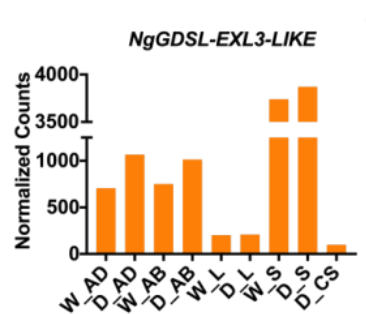

J

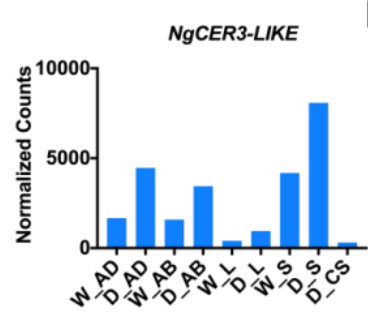

$\mathrm{N}$

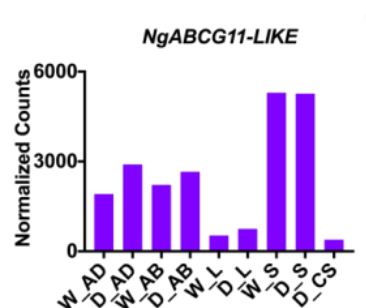

C

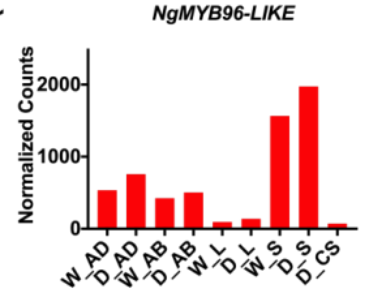

G

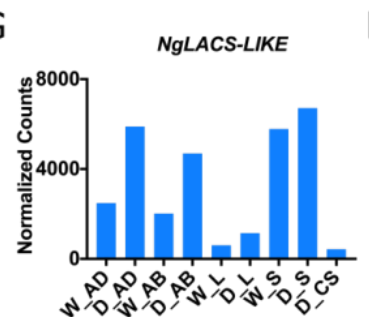

K

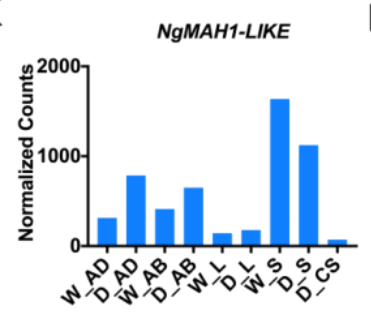

0

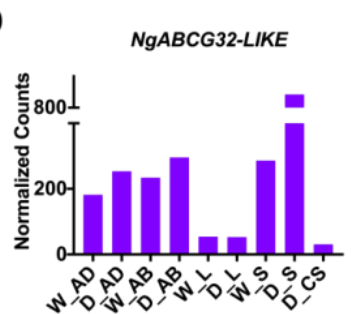

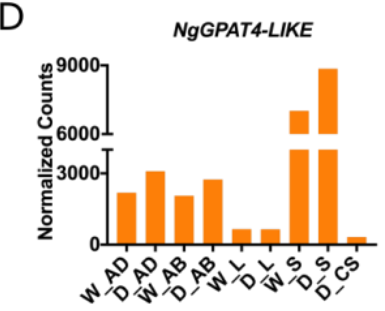

$\mathrm{H}$

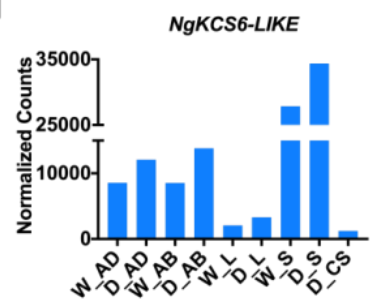

L

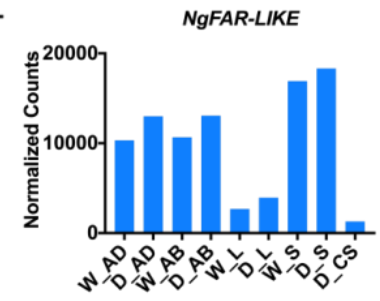

P

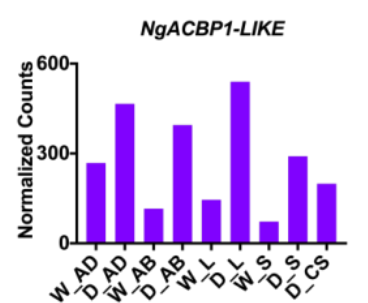

Figure 2. Epidermis enriched and drought-induced expression of $N$. Glauca genes showing homology to known cuticular lipids genes from other species. For each sample, three biological replicates of each tissue, grown either under well-watered conditions or drought treated, were collected and pooled to reduce variance though not increasing the number of sequenced samples. The different genes are color coded according to their function; transcription factors in red (A-C), cutin synthesis genes in orange (D-F), wax synthesis genes in blue (G-M), and transporter genes in purple (N-P). AD- adaxial epidermis 
tissue; AB- abaxial epidermis tissue; L- whole leaf tissue; S- stem epidermis tissue; CScomplete stem; W- watered plants; D- drought treated plants.

\section{Cuticular lipids mutants display diverse surface and morphological phenotypes}

Of the 16 genes targeted, nine exhibited visual surface related phenotypes at the T0 generation (Fig. 3 and Fig. S1). These included glossy leaves and stems and fused anthers in the kcs6 mutant alleles, glossy leaves and stems in the far and cer 1 mutants and glossy leaves alongside waxy stems in cer3 mutants (Fig. 3). The abcgl1 mutant, the only line that never flowered (even over two years) possessed small, glossy, crinkled, brittle leaves. Both cyp86a22 and abcg32 showed elongated and malformed leaves, while gpat4 displayed elongated leaves and leaf fusions. Finally, the lacs mutants displayed both a glossy phenotype and leaf deformities (Fig. S1). Sequencing the amplicons covering the targeted editing sites revealed a wide range of mutations; from single base pair insertions or deletions, deletion of a triplet coding for a single amino acid to a 200 bp deletion (Fig S2-S10). First generation plants (i.e. T0) had both homozygous and heterozygous mutations, and frequently carried two different mutations on their two chromosomes, and not merely two identical mutations. 


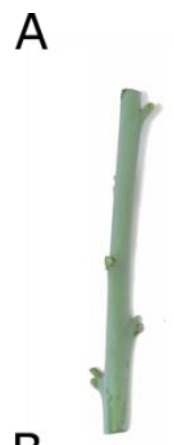

B

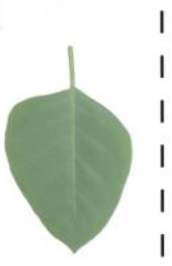

WT

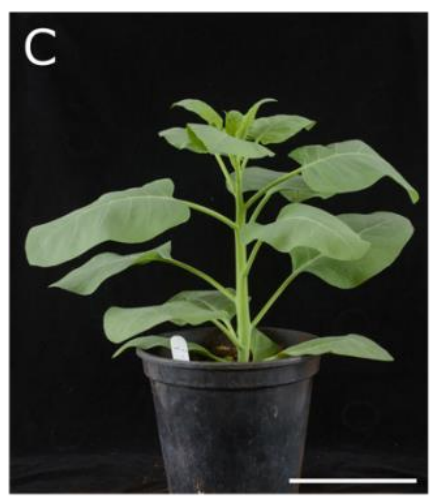

$\mathrm{F}$

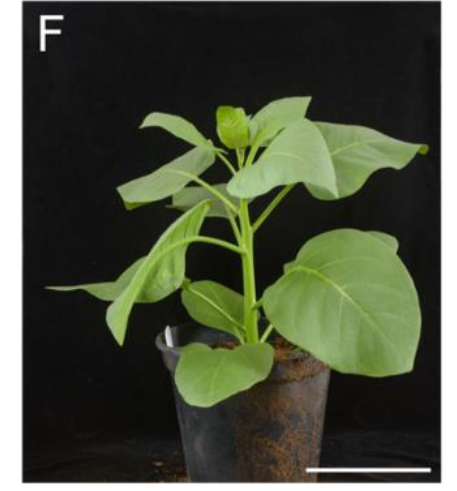

I

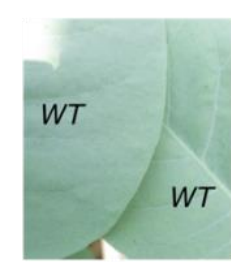

WT

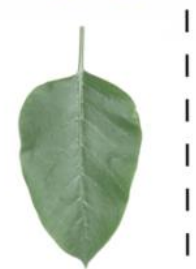

far

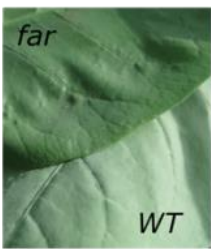

far
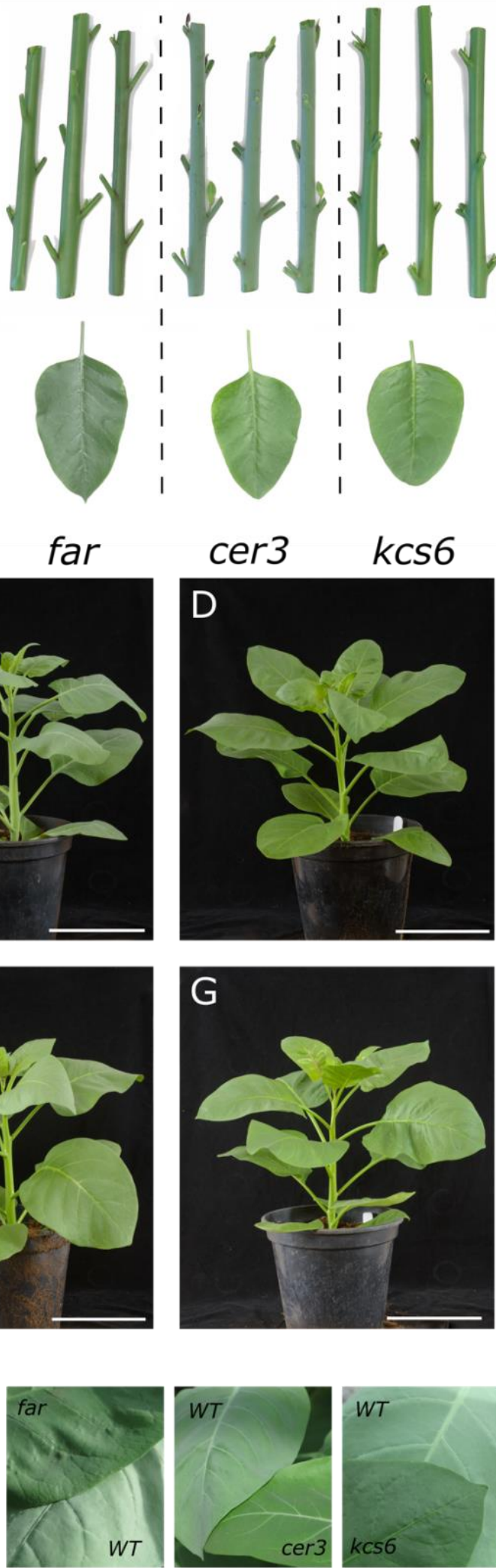

cer3

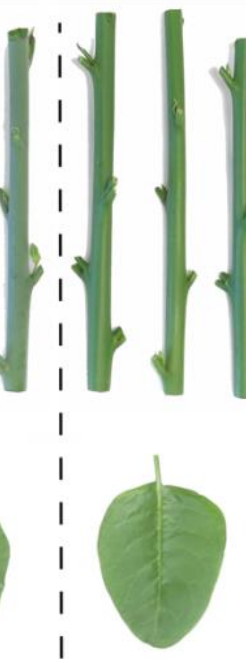

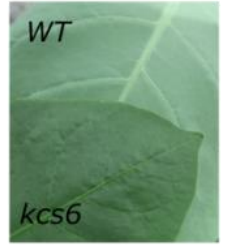

kcs 6
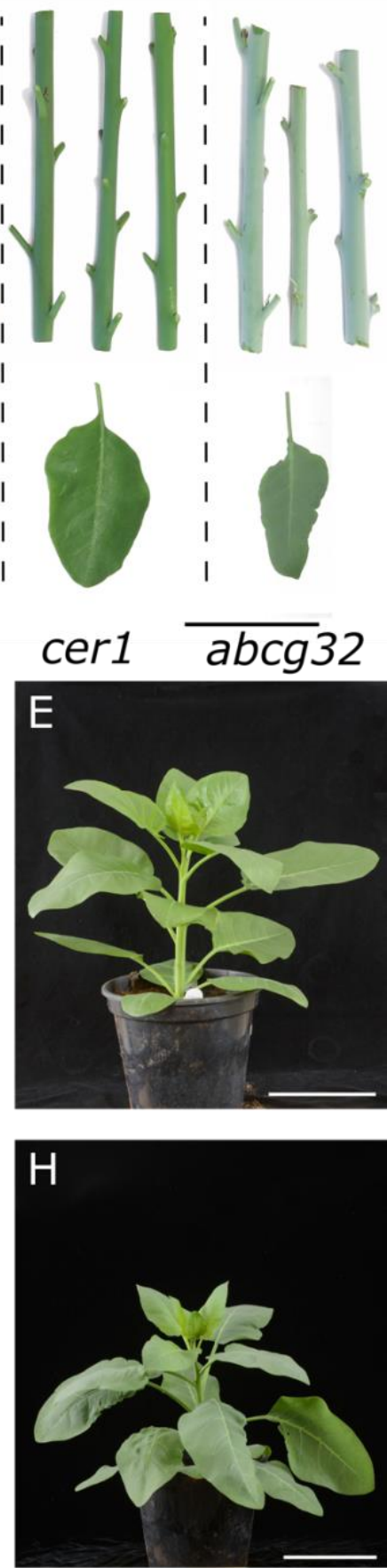

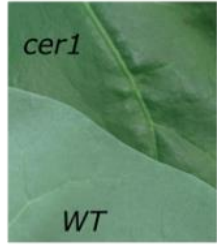

cer1 abcg32

Figure 3. Plant surface phenotypes of mutant plants analyzed in this study. (A)

Representative stems of wild type (WT) and the five independent mutant lines investigated in-depth. (B) Representative leaves of WT and mutants' leaves. Bar $=5 \mathrm{~cm}$. (C-H) 
representative whole plants Bar=15cm. C, WT; D, far; E, cer3; F, kcs6; G, cerl; H, abcg32. (I) images of WT leaves and those of mutants alongside.

\section{Surface wax composition and crystal morphology in wax genes mutants}

Out of the nine mutants, we focused the study on four wax metabolism genes, as well as the abcg32 mutant that served as a control as it is affected in cutin monomer transport. We next extracted leaves' epicuticular wax and analyzed its composition using gas chromatography mass spectrometry (GC-MS). Wax composition in N. Glauca is typically dominated by a $\mathrm{C}_{31}$ alkane (92\% according to Mortimer et al., 2012) along with $\mathrm{C}_{33}$ alkanes, $\mathrm{C}_{24}, \mathrm{C}_{26}$ and $\mathrm{C}_{28}$ fatty alcohols and smaller amounts of a $\mathrm{C}_{26}$ aldehydes (as well as trace amounts of other alkanes, alcohols and aldehydes). Mutations in the different genes had a drastic effect on epicuticular wax composition (Fig. 4). kcs6 mutant leaves displayed almost complete reduction in all wax

components with a chain length above 26 carbons making them nearly free of alkanes (Fig. 4A-B). The same leaves accumulated shorter chain length waxes such as $\mathrm{C}_{18}, \mathrm{C}_{20}, \mathrm{C}_{22}$ and $\mathrm{C}_{24}$ alcohols (Fig. 4C-F). Leaves of the cerl mutants showed massive reduction in abundance of all alkanes (Fig 4A-B) mirrored by a significant increase in $\mathrm{C}_{26}$ alcohols (Fig. 4E). cer3 mutants had a significant reduction (approximately 80\%) in their $\mathrm{C}_{31}$ wax load. In the same mutant leaves, the $\mathrm{C}_{33}$ alkane showed a trend of increase that was significant in one independent line (Fig. 4A-B). The far mutants displayed extremely reduced fatty alcohol content (Fig. 4C-F) contrasted by a trend of increase in their alkane load (Fig 4A-B). abcg32 mutants' wax composition was similar to WT, with only a trend of reduction in $\mathrm{C}_{31}$ alkane load (Fig. 4A) and a significant increase in $\mathrm{C}_{26}$ alcohols in abcg32-18 and an increase in $\mathrm{C}_{28}$ alcohols in both abcg32-6 and abcg32-18 (Fig. 4E-F). 
A

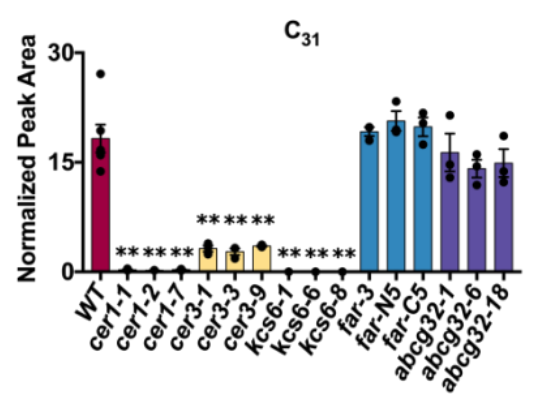

C

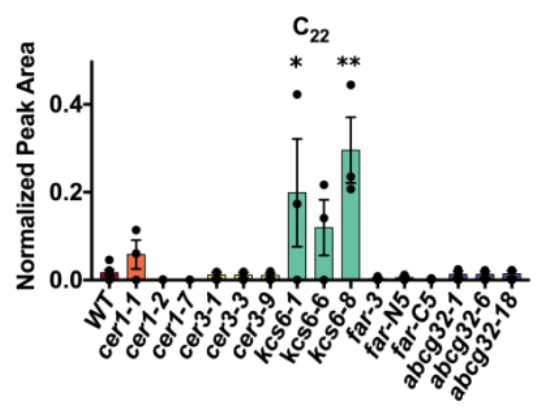

$\mathrm{E}$

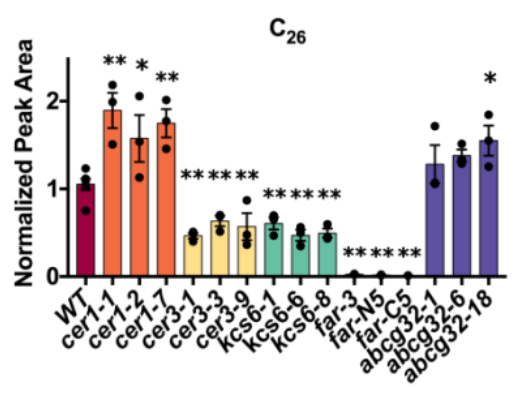

B
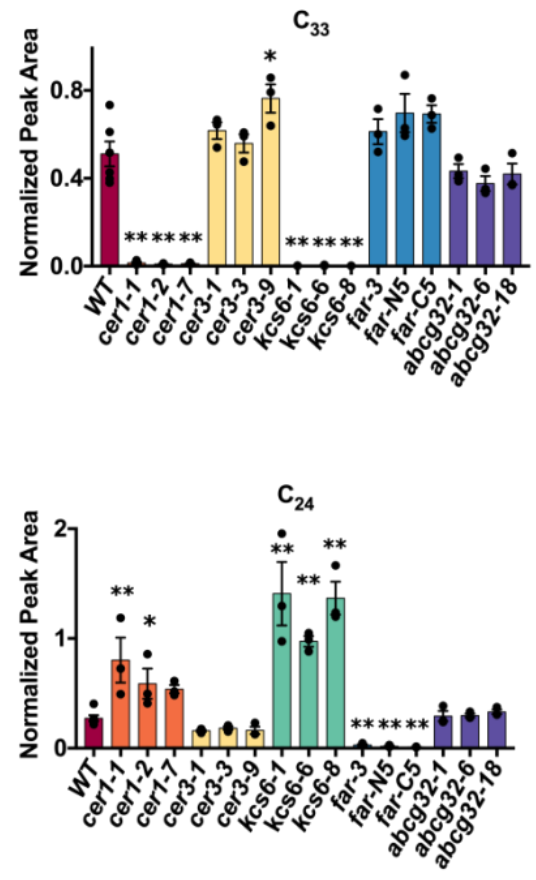

$\mathrm{F}$

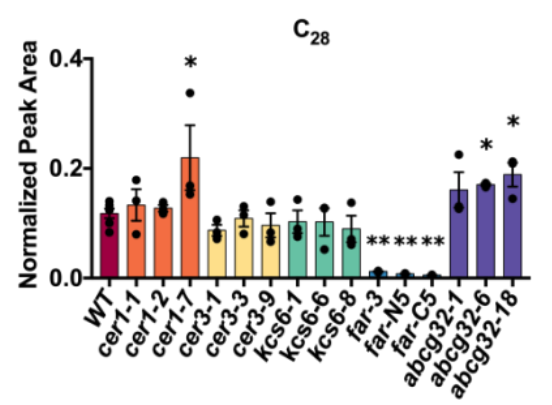

Figure 4. Relative abundance of major wax components in N. glauca leaves. (A-B) Alkanes and (C-F) primary alcohols. Numbers indicate carbon chain length. Independent mutant alleles of the same gene are indicated by the same color. WT, n=6; all other lines $n=3$. *= $\mathrm{p}<0.05$ and ${ }^{* *}=\mathrm{p}<0.01$ as determined in a student's $\mathrm{t}$ test. Bars represent standard error. Wax components were extracted in chloroform, derivatized and quantified by GC-MS

Alterations in composition of epicuticular waxes had a strong effect on wax crystal morphology as visualized using cryo-SEM (Fig. 5). The typical wax crystals of $N$. Glauca leaves are of dense rodlets (Fig. 5A). In cerl leaves, a thin layer of flakes replaced wax rodlets normally found in WT leaves (Fig. 5B). Leaves of cer3 displayed crystal morphology similar to that of the WT leaves, however, these crystals were reduced in size and more sparsely distributed on the leaf surface (Fig. 5C). Leaf wax of kcs6 lost its rodlet-like morphology and instead appeared as structured lines of vertically positioned membranous platelets (Fig. 5D). The far leaves exhibited large vertical sharp-edged plates, which were 
sparsely distributed on the leaf (Fig. 5E). Finally, the abcg32 mutant wax crystals appeared normal as expected from its almost unaltered wax composition (Fig. 5F).

A
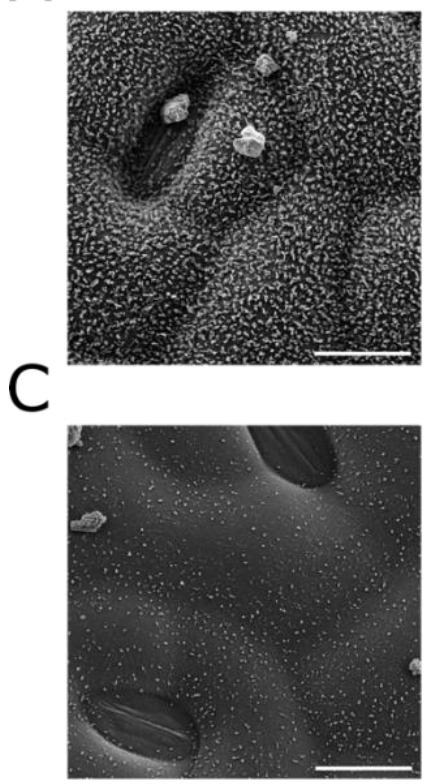

$\mathrm{E}$

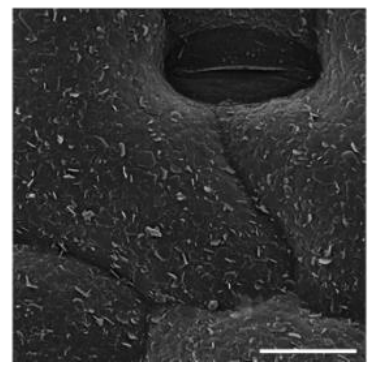

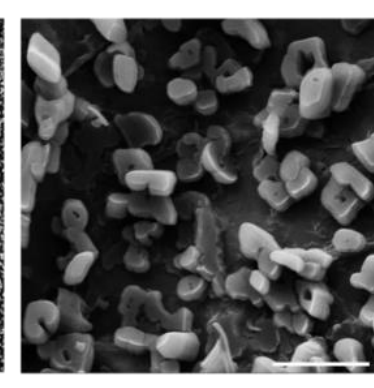
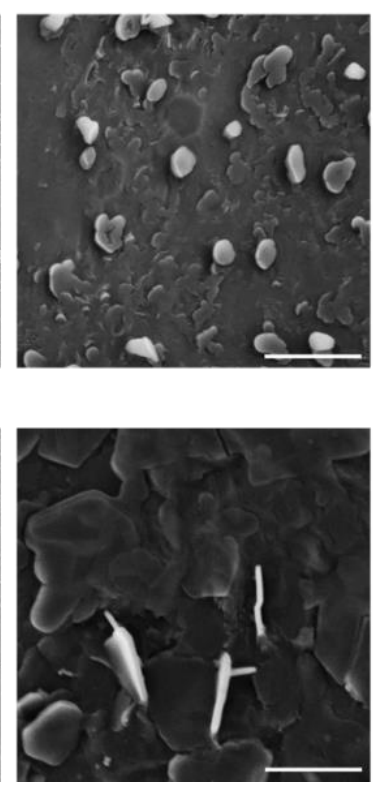

B
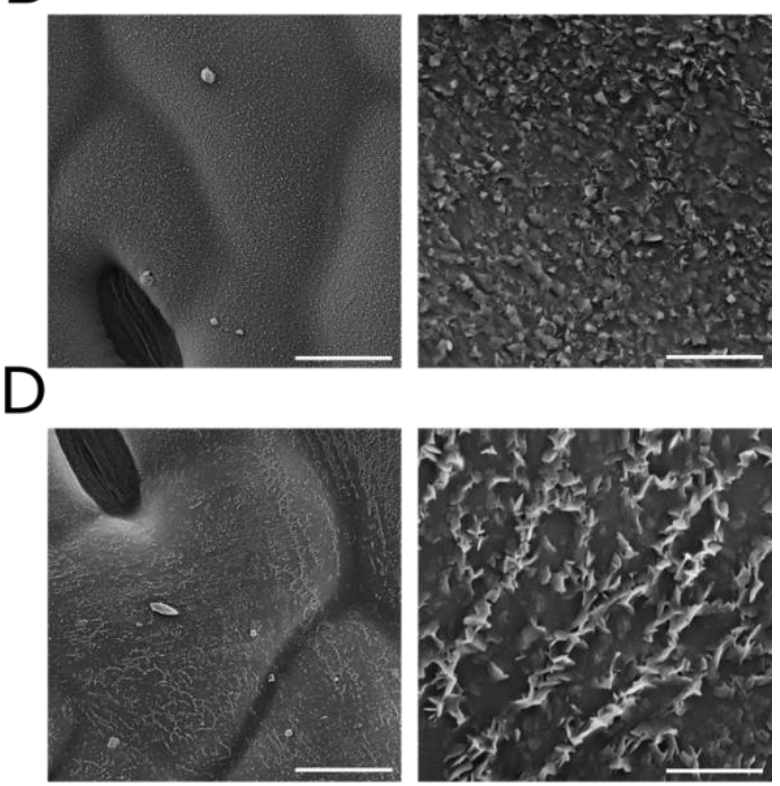

$\mathrm{F}$
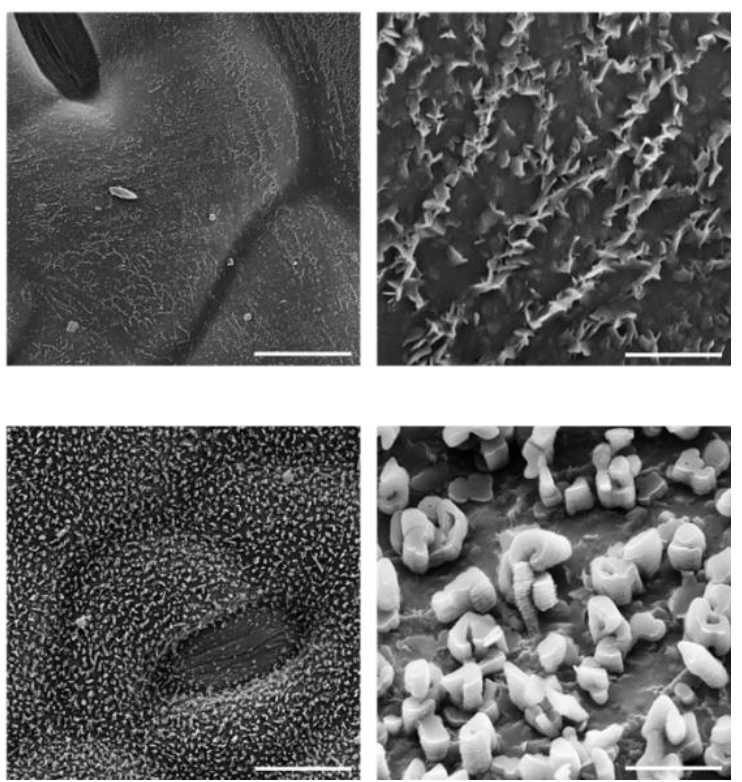

Figure 5. Cryo SEM images of wax crystal dispersion and structure on the adaxial side of mutant plants. (A) WT leaf; (B) cerl leaf; (C) cer3 leaf; (D) kcs6 leaf; (E) far leaf; (F) abcg32 leaf. For each line, a magnification of $\mathrm{x} 2500$ with a $20 \mu \mathrm{M}$ bar is shown on the left and one magnified to $\mathrm{x} 25,000(2 \mu \mathrm{M}$ bar $)$ on the right.

\section{Cutin composition of wax metabolism mutants}

Wax metabolism and cutin metabolism share some common enzymes and precursors. We therefore examined cutin composition in the same lines analyzed for epicuticular wax (Fig. 6). Here the impact of mutations was far less drastic as compared to their effect on wax composition - not coming close to a reduction percentage of the stronger wax mutants. This was not the case for the cerl lines showing significantly altered loads of several cutin monomers, and reaching a reduction of $\sim 64 \%$ in methyl caffeate abundance (Fig. 6A-H). Furthermore, when we analyzed the data per chemical class, we found that cerl mutants had significantly lower fatty acid load in two out of three lines (Fig. 6B), but a significantly 
higher levels of $\omega$-hydroxylated fatty acids (Fig. 6C). Mirroring their wax composition, all three cerl lines had significantly higher content of very long chain fatty alcohols. Interestingly, cerl lines also had reduced total phenol content in their cutin (Fig. 6E).

A

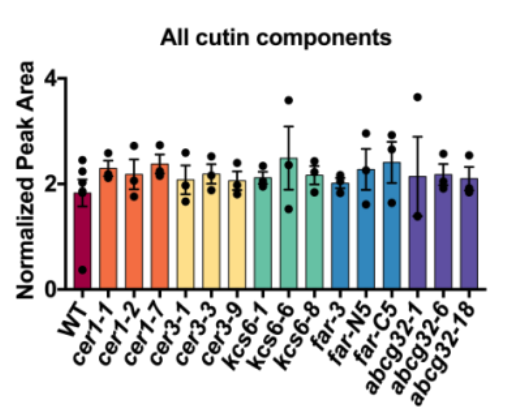

C

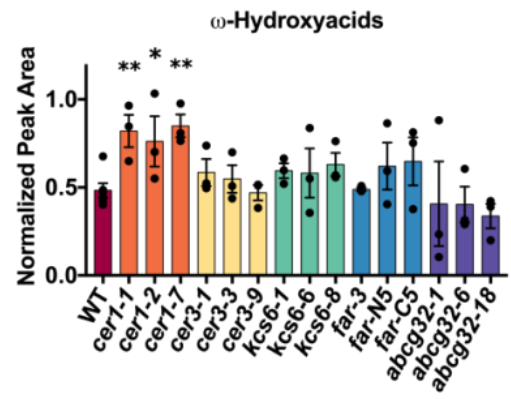

$\mathrm{E}$

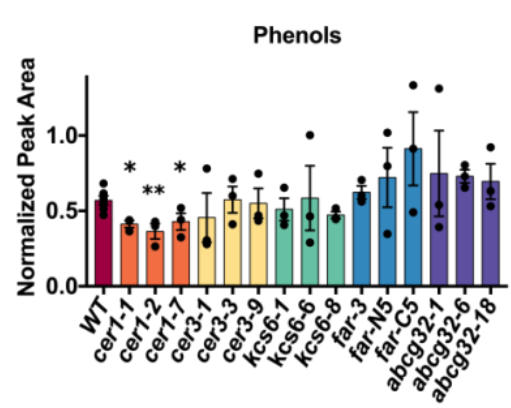

G

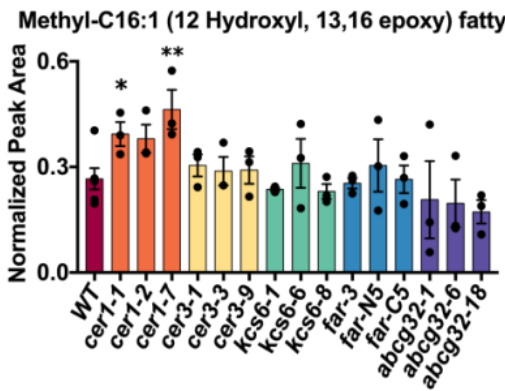

B

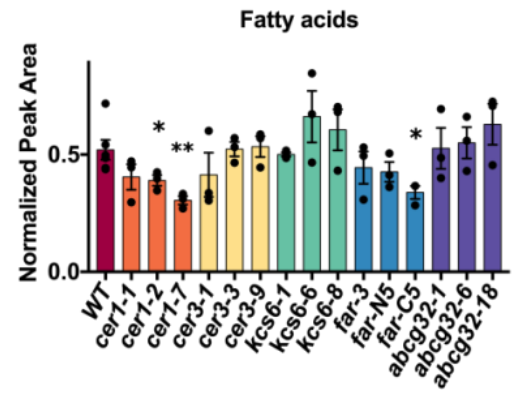

D

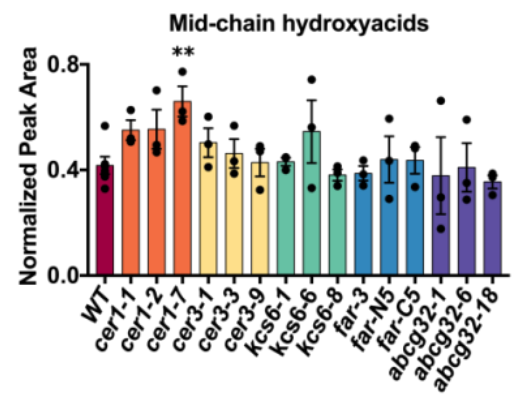

$\mathrm{F}$

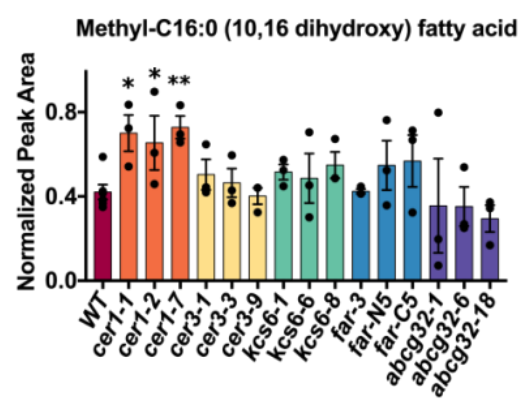

$\mathrm{H}$

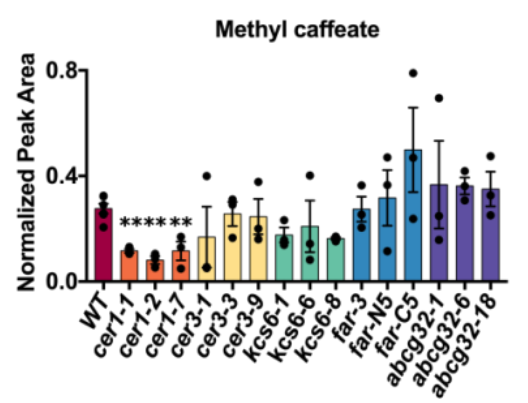

Figure 6. Relative abundance of cutin and its monomers in mutant lines. (A-E) Analysis of main cutin monomer classes. (F-H) Analysis of most abundant cutin monomers. WT, $\mathrm{n}=6$; all other lines $\mathrm{n}=3 . *=\mathrm{p}<0.05$ and $* *=\mathrm{p}<0.01$ as determined in a student's $\mathrm{t}$ test. Bars represent standard error. All samples were quantified using GS-MS following delipidation, transesterification and derivatization. 


\section{Reduction in alkane abundance increases cuticular water loss drastically}

We next examined how changes in chemical composition affected the physiological state of mutant plants by analyzing cuticular water loss. Leaves were detached from plants and weighed every two hours once reaching the linear weight loss stage. Out of the five mutant genotypes, the cerl mutants were most strongly affected, showing a three times higher water loss rate (Fig. 7A). The kcs6 mutant leaves also showed a higher water loss rate whereas abcg32 leaves displayed a slight and yet significant increase. Surprisingly, of the cer3 mutant lines only cer3-1 had a significantly elevated water loss rate. In contrast, two of three far lines lost water significantly slower as compared to WT leaves.

A

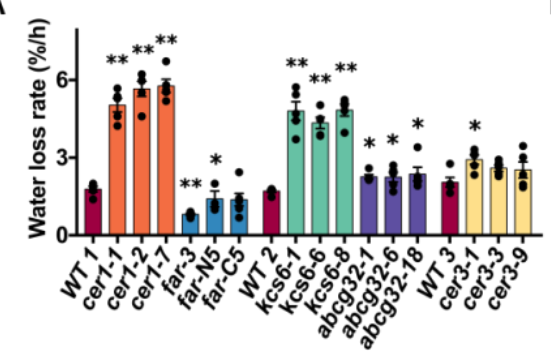

C

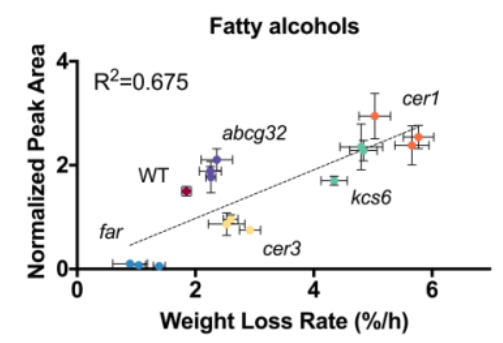

$\mathrm{E}$

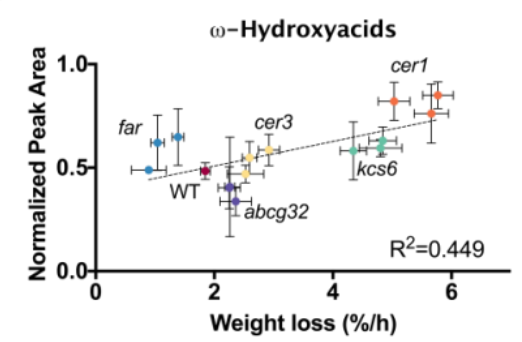

B
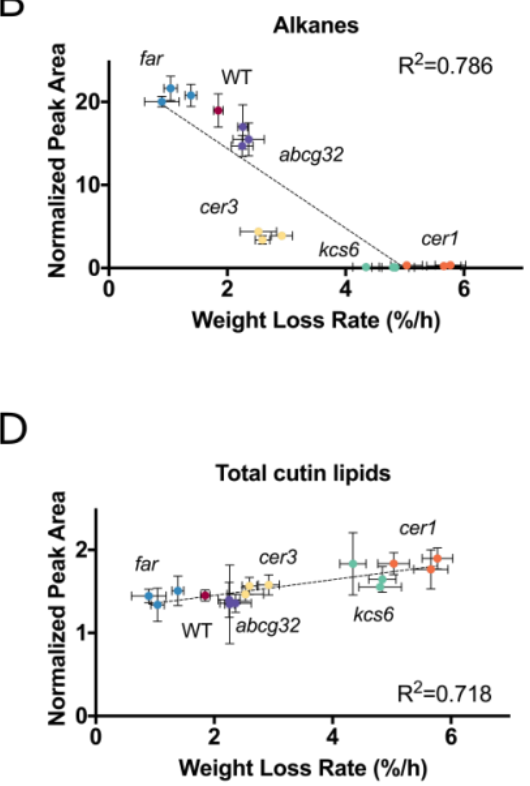

$\mathrm{F}$

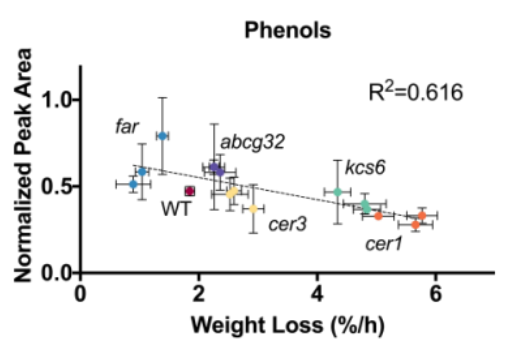

Figure 7. Cuticular water loss rate and its correlation to different wax and cutin components. (A) Cuticular water loss rate. The experiment included three assays in consecutive days each with its own WT samples (WT1 to WT3). Mutant alleles of the same gene are indicated in the same color. $*=p<0.05 ; * *=p<0.01$ determined in a student's $t$ test; $n=5$. Bars represent standard error. Correlation between water loss rate and (B) sum of all alkane wax components, (C) sum of very long chain fatty alcohols, (D) sum of all lipidic components of the cutin monomers; (E) sum of $\omega$-hydroxylated fatty acids of the cutin monomers; (F) sum of all phenols of the cutin monomers. $\mathrm{R}^{2}$ indicated in each correlation graph is generated 
from average water loss rate and average abundance of the different wax and cutin components in in the same independent mutant line, obtained in separate experiments.

Next, we plotted water loss rate against the average abundance of wax and cutin components in independent mutant lines (Fig. 7B-F). We found a correlation with an $\mathrm{R}^{2}$ of 0.786 between water loss rate and total alkane content. Yet, cer3 leaves lost water at a slower rate than their alkane content would predict while cerl plants lost water at a faster rate than that predicted by the linear regression (Fig. 7B). When cutin components were plotted against water loss, a positive correlation $\left(\mathrm{R}^{2}=0.718\right)$ was found with total cutin lipids, although the differences in the cutin component abundance was far smaller between the different lines compared to those in wax components (Fig. 7D). In contrast, phenols found in the cutin fraction $\left(\mathrm{R}^{2}=0.616\right.$; Fig. $\left.7 \mathrm{~F}\right)$, and especially methyl caffeate $(\mathrm{R} 2=0.7)$ were negatively correlated with water loss. Although the role of the phenolics is not clear to us, these results highlight that alkanes in the epicuticular wax fraction play an important role in preventing cuticular water loss.

\section{Wax has little effect on water loss pre-drought, but alkanes are essential for recovery from drought}

The major effect on cuticular water loss rates due to changes in epicuticular waxes led us to hypothesize that under well-watered conditions plants with greatly reduced alkane abundance would transpire at higher rates and be more susceptible to drought. We tested this hypothesis using a weighing lysimeter system, in which plants are weighed every three minutes and in combination with environmental measurements (e.g. temperature, humidity and light intensity) performed in parallel, transpiration, stomatal conductance $\left(\mathrm{g}_{\mathrm{s}}\right)$, biomass accumulation, water use efficiency (WUE) and drought response can be extrapolated. The first two experiments performed with this system in the summer and winter seasons, involved two and three different mutant alleles of cer3, respectively. To our surprise, in the first experiment (during the summer), cer3 plants had an almost identical daily transpiration compared to WT ones (Fig. S11A). In the 'winter' experiment, we observed a trend of cer3 transpiration starting to be reduced compared to WT as plants grew and their transpiration rate increased, although this trend did not reach significance prior to the onset of drought (Fig. S12A). Furthermore, cer3 biomass accumulation was not impaired in both experiments (Fig. S11g, S12G), as was their WUE (Fig. S11J, S12I). Plants that respond to drying soil by closing their stomata at a higher soil water content ("theta point") are more likely to show 
drought tolerance. Drought response in these two experiments was therefore measured by evaluating this theta point. In the 'summer' experiment, cer3 lines had a theta point similar to WT (Fig. S11K), whereas in the 'winter' experiment, their theta point was at a significantly higher soil water content (Fig. S12J). The results indicated that cer3 plants (displaying approximately $80 \%$ reduction in $\mathrm{C}_{31}$ alkane) were not negatively affected by their reduced wax load both under well-watered and drought conditions.

The findings described above prompted us to examine whether plants mutated in additional genes that severely affect wax composition would behave in a similar manner. To answer this question, we performed an additional experiment during the spring. Following the previous experiments, we reasoned that during this season we could enjoy the advantages of both the 'summer' experiment in which plants grow at a fast rate and the vapor pressure deficit (VPD) and light intensity are relatively stable and the 'winter' experiment in which plants are less susceptible to diseases. In this 'spring' experiment, we placed three different mutant alleles of either cerl, kcs6 or far on the lysimetric system alongside three lines of WT plants. Similar to the cer3 results under well-watered conditions there was no significant difference between the mutants and WT plants in daily transpiration (Fig. 8A), transpiration rate (Fig. 8D) plant growth rate (Fig. 8G), and water use efficiency (Fig. 8J).

Once we exposed well-watered plants to drought and recovery (i.e. re-watering), we detected a markedly different response in the wax mutants. The cerl mutant lines' theta point was significantly lower than WT, while kcs6 and far were similar to WT (Fig. 8K). This led to cerl plants having a significantly higher transpiration rate throughout long periods of the day, that was not seen in the other mutant lines (Fig. 8E). Though the increase in cerl's transpiration rate is significant, the effect size is relatively small, and reached a $\sim 20 \%$ increase in transpiration rate during the afternoon hours. This was not the case following resuscitation. At this phase, the daily transpiration of far plants was significantly higher as compared to WT plants that exhibited significantly greater transpiration than cerl and kcs6 (Fig. 8C, F). These differences rose with time up to the sixth day following resuming irrigation; far plants transpired 533ml a day on average, WT $262 \mathrm{ml}$, kcs6 $50 \mathrm{ml}$ and cerl transpired only $36 \mathrm{ml}$ on average during that day (Fig. 8C). These results were mirrored by the transpiration rate (Fig. 8F), plant growth rate (Fig. $8 \mathrm{H}$ ) and shoot dry biomass (when the experiment ended; Fig. 8I). The underlying reason for these drastic differences was evident when we examined the plants once completing the experiments (Fig. S13). While all far plants retained their original leaves, some WT plants were left with leaves and others not. In a vast majority of the cerl and kcs6 plants leaves present at the time of drought initiation 
dried and the transpiration was from new leaves, which started growing from axillary buds upon resuscitation

(Fig.

S13).

A

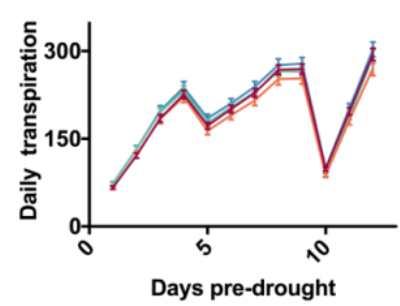

D

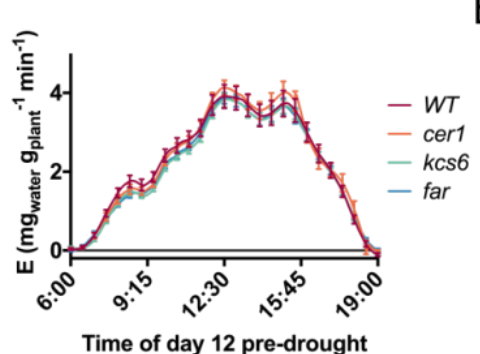

G

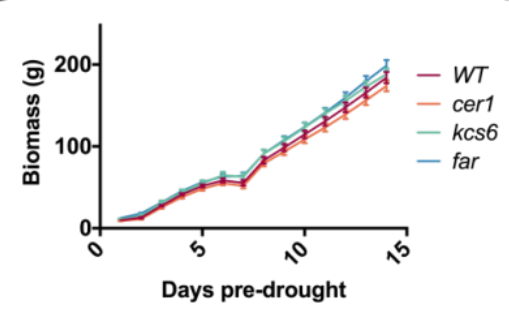

]

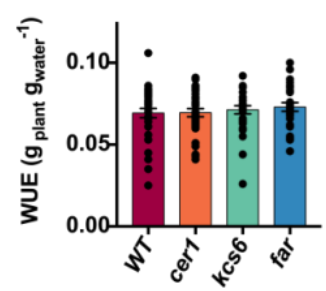

B

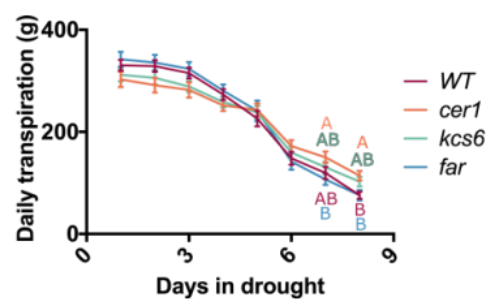

E

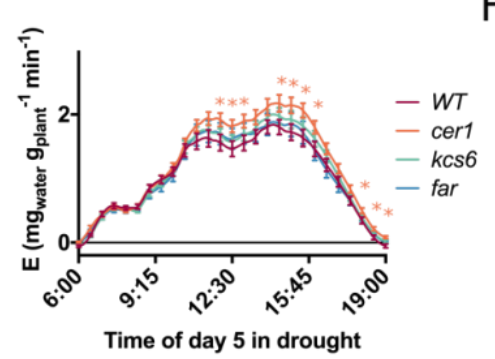

$\mathrm{H}$

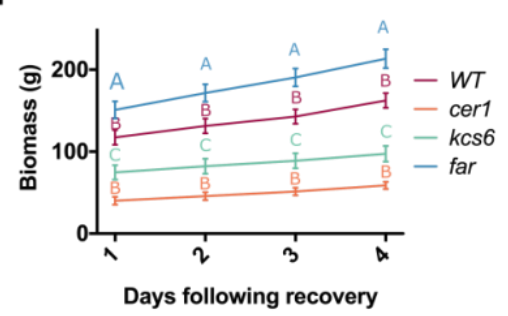

$\mathrm{K}$

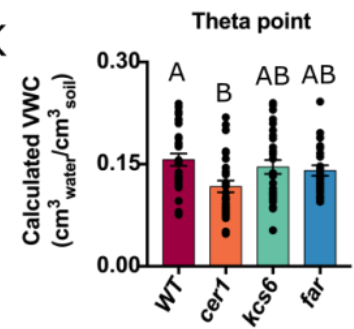

C

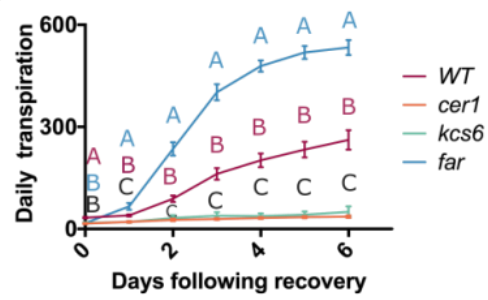

$\mathrm{F}$

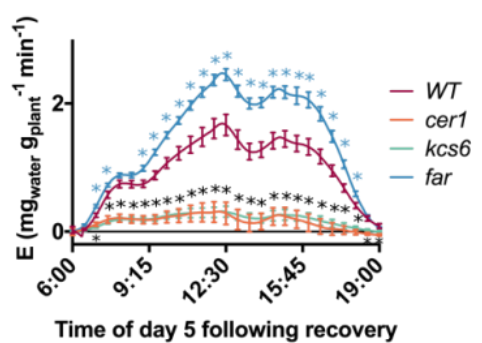

I

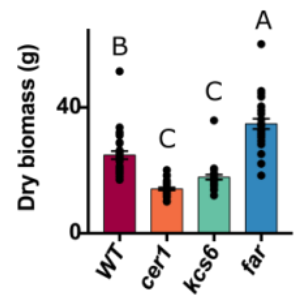

Figure 8. Multi-parameter analysis of mutant plants assayed in a lysimeteric system and assayed following full irrigation, drought and recovery. (A) Pre-drought daily transpiration. (B) Daily transpiration following drought initiation and prior to resuscitation. (C) Daily transpiration following recovery. Since each individual plant received full irrigation three days after it transpired below the $20 \%$ daily transpiration threshold (see materials and methods), the days in recovery unlike those pre-drought and during drought are different for each individual plant, and are averaged according to the number of days past irrigation renewal per plant. (D) Transpiration rate during day 12 prior to drought induction. Measurements were taken every $3 \mathrm{~min}$, though error bars are only shown every $30 \mathrm{~min}$. (E) Transpiration rate during the fifth day of drought. (F) Transpiration rate during the fifth day after plant resuscitation, averaged from different dates for each plant according to its date of recovery. (G) Plant biomass during the period prior to drought induction. (H) Plant biomass 
during the four days before the experiment ended, at which time all plants had been resuscitated. (I) Dry shoot biomass at the experiments end. (J) WUE as calculated predrought. (K) "Theta point" volumetric water content at which plants began reducing their transpiration rate in response to drying soil. All data is the average of three independent mutant alleles used in this experiment, including WT which had three independent lines grown first in tissue culture prior to the transfer to the greenhouse as performed with all mutant lines. The following amount of lines were analyzed: WT $n=28$ to 33 ; $\operatorname{cer} 1 \mathrm{n}=27$ to 31 ; kcs6 $\mathrm{n}=25$ to 29 ; far $\mathrm{n}=24$ to 26. Different letters indicate significance of $\mathrm{p}<0.05$ as determined in a Tukey HSD test. Asterisks represent significance of $p<0.05$ as determined in a student's t test. Asterisks were placed above standard error lines (bars), though points are significant for every point measured between the bars.

\section{Drought followed by resuscitation leads to stem cracking}

Towards the completion of the weighing lysimeters experiment, we detected an intriguing phenotype, which to the best of our knowledge has not been reported in the context of wax deficiency. Sixteen-out of 27 kcs 6 lines derived from all three independent mutant alleles displayed severe cracks along their stems penetrating the xylem and deep into the stem pith (Fig. 9A-B). Intriguingly, these cracks even led to water dripping freely out of the xylem and out of the cracks (Fig. 9A). Besides the kcs6 plants, there was one occurrence of stem cracking in a cerl-1 plant indicating that the occurrence in kcs6 plants was not an isolated event. When investigating the reasons for the kcs6 stem phenotype we found that the $11 \mathrm{kcs} 6$ plants with intact stems whose non-cracking phenotype was not due to technical reasons had a similar biomass and theta point compared to the plants with cracked stems at the onset of drought (Fig. 9E; Fig. 9F). While all 16 cracked plants resuscitated during three days, from the $13^{\text {th }}$ day of drought to the $15^{\text {th }}$ day, the uncracked plants were resuscitated between the $10^{\text {th }}$ to the $18^{\text {th }}$ day in drought. When examining the $11 \mathrm{kcs} 6$ plants with undamaged stems we found that they reduced their volumetric water content (VWC) at a slower rate than those with cracked stems, though this difference was not significant on a point measurement basis (Fig. 9G). Thus, as is seen from the wider range of drought duration prior to recovery, plants that depleted their water and resuscitated rapidly as well as those that transpired less and were exposed to drought more gradually did not experience stem cracking. 
A
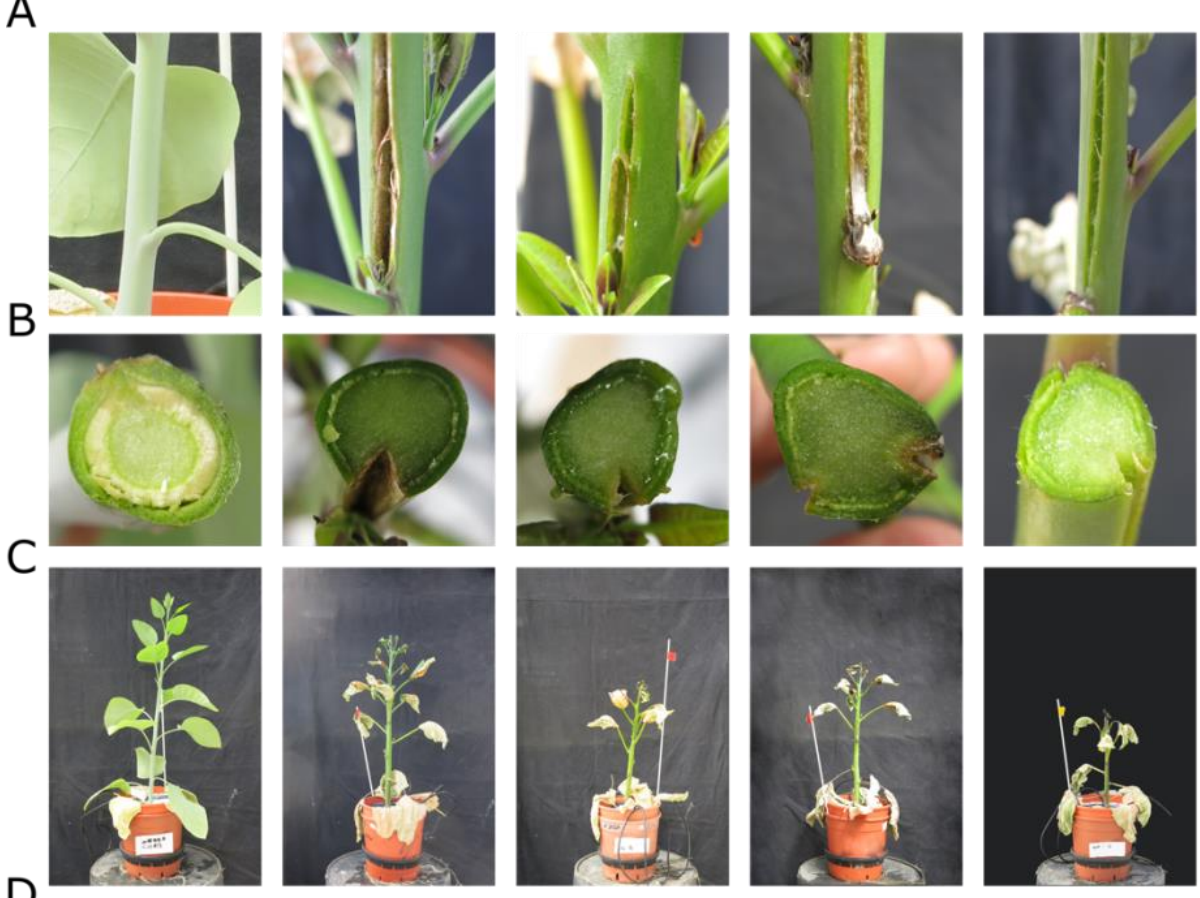

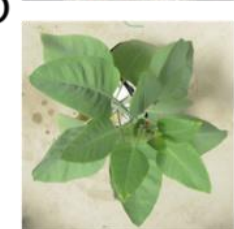

WT

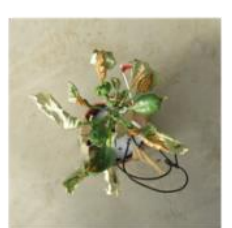

$k \operatorname{cs} 6-1$

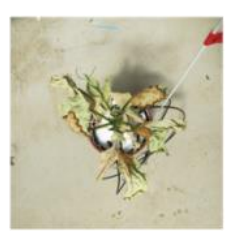

kcs6-6

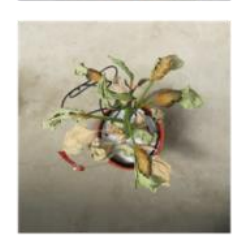

$k \operatorname{cs} 6-8$

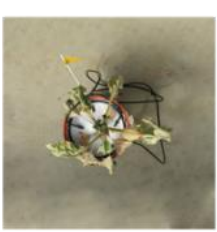

$\operatorname{cer} 1-1$

$\mathrm{E}$

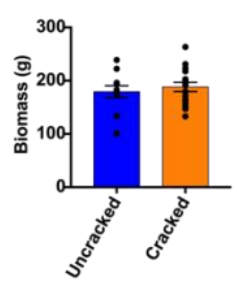

$\mathrm{F}$

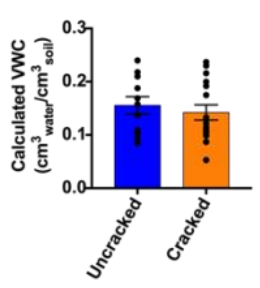

G

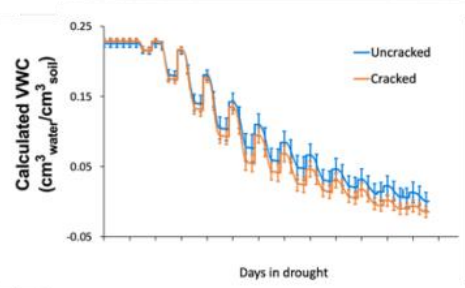

Figure 9. The stem cracking phenomenon. (A) Images of cracked stems phenotypes in representative plants from the three $k c s 6$ independent mutant alleles, a cer 1 plant displaying a similar phenotype and a representative WT stem. (B) Images of cut stems showing the radial formation of the crack through the xylem and into the stem pith cells. (C) Images of whole plants at the experiments end. (D) Whole plants photographed from above and displaying differential drought response. (E) Biomass of plants with or without cracked stems $(k c s \sigma)$ measured during the last irrigated day prior to drought. (F) Volumetric water content of kcs6 plants in which no stem cracking was observed compared to those in which stems cracked. Plants included in this analysis were of similar size at drought onset. (G) VWC in pots of cracked and uncracked $k c s 6$ plants as drought commenced. Cracked $\mathrm{n}=16$; uncracked $\mathrm{n}=11$.

\section{Stomatal aperture and development do not compensate for elevated cuticular water loss}

We examined whether the discrepancy between high leaves cuticular water loss and normal transpiration rate under well-watered conditions was related to a reduced stomatal density, 
size or aperture, leading to the elevated cuticular water loss being compensated for by reduced stomatal water loss. However, all mutants had similar stomatal density (Fig. S14A, D) and sizes (Fig. S14B,E) compared to WT, and even those insignificant differences in density were compensated for by larger stomata; i.e. plants with a trend of less dense stomata had a trend of larger stomata as well. Abaxial stomatal aperture (Fig. S14C) was the only parameter where we found a significant difference, with cerl having a larger stomatal aperture. Thus, unlike our original hypothesis, cuticular water loss was not compensated for by stomatal aperture or morphology.

\section{The glossy phenotype of wax mutants does not significantly affect light response and plant fitness}

Despite extreme differences in their wax composition, cerl, cer3, kcs6 and far mutants, all displayed a glossy leaf appearance. To examine if this phenotype affects photosynthetic efficiency, we examined RUBISCO efficiency ( $\mathrm{Vc}_{\max }$ ) and maximal electron transport rate $\left(\mathrm{J}_{\max }\right)$ by monitoring carbon assimilation as sub-stomatal $\mathrm{CO}_{2}$ concentration was raised $(\mathrm{A} / \mathrm{Ci}$ curves). The cer 1 mutant lines appeared different from all other mutants and the WT as their A/Ci curve plateaued much earlier than all other genotypes (Fig. 10A). Although the $\mathrm{J}_{\max }$ of cerl plants was not significantly lower (Fig. 10C), their $\mathrm{Vc}_{\max }$ was significantly reduced (Fig. 10B). cerl's wax composition is similar to $k c s 6$, a fact that leads to very similar responses in A

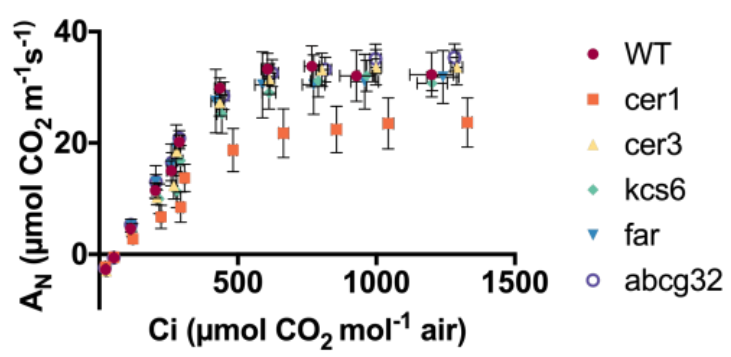

B

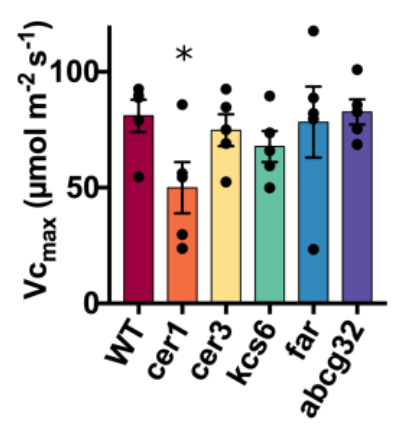

C

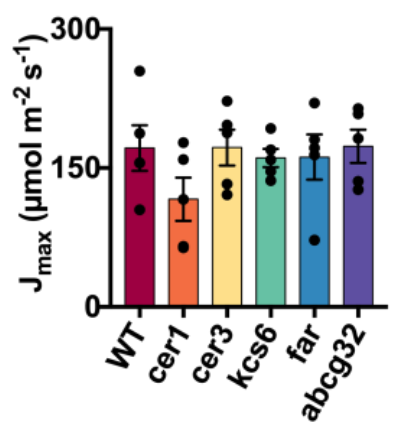

Figure 10. Photosynthetic efficiency of WT and four wax biosynthesis mutant lines as analyzed by $\mathrm{A} / \mathrm{Ci}$ curves (A) Average carbon assimilation with rising $\mathrm{Ci}$ concentrations. (B) $\mathrm{Vc}_{\max }$ indicating RUBISCO efficiency. (C) $\mathrm{J}_{\max }$ indicating maximal electron transport rate. Asterisks indicate a significance of $\mathrm{p}<0.05$ as determined in a student's t test. $\mathrm{N}=5$. 
assays where wax composition is the underlying factor. However, cerl is the only mutant with altered cutin, leading us to suggest that the differences seen in the A/Ci curves were the result of altered cutin and not wax load or composition.

We next asked if the glossy phenotypes of wax mutants had a significant impact on light response and consequently plant fitness. Thus, we exposed plants of each genotype to 12 rising light intensities and recorded carbon assimilation and stomatal conductance (Fig. 11A and Fig. 11B). No plants reached photoinhibition along these points despite reaching 2200 $\mu \mathrm{E}$ (while plants were grown at a light intensity of $100 \mu \mathrm{E}-200 \mu \mathrm{E}$ ). Similar to the $\mathrm{A} / \mathrm{Ci}$ curves results, cerl mutants were the only plants displaying an altered phenotype exhibiting reduced carbon assimilation at five points of the dozen light intensities measured (Fig. 11A). These results demonstrate that light reflectance by wax crystals does not alter photosynthetic capacity under non-stressed conditions.
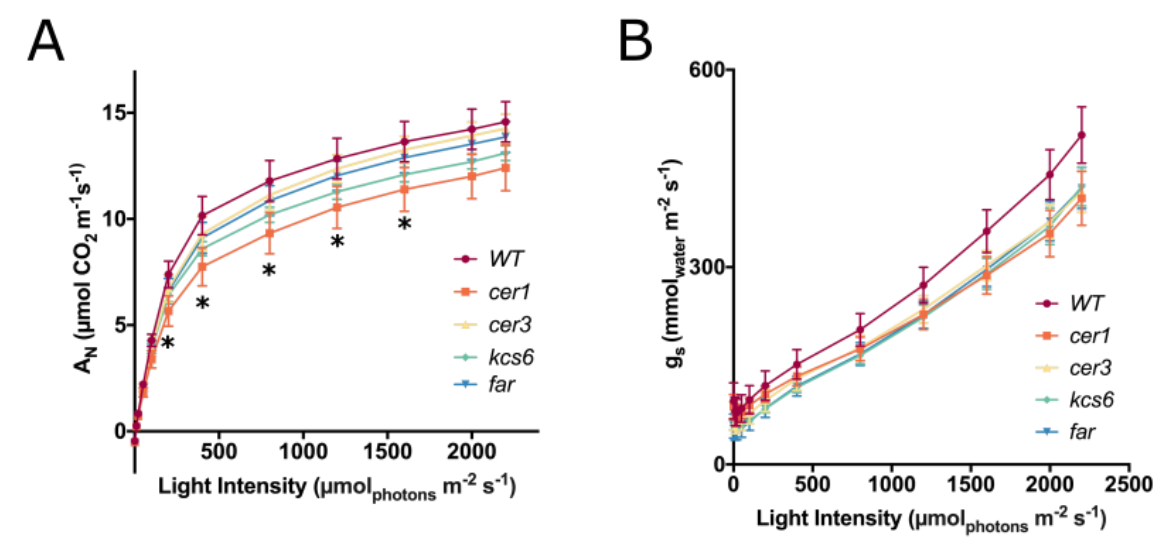

Figure 11. Effects of wax deficiency on gas exchange in response to rising light intensity. (A) Carbon assimilation. (B) Stomatal conductance. Asterisks indicate a significance of $\mathrm{p}<0.05$ as determined in a student's $\mathrm{t}$ test. $\mathrm{N}=9$. 


\section{Discussion}

\section{Tree tobacco in cuticular lipids research}

For many years, cuticular lipids research has been largely performed in Arabidopsis thaliana (Lee and Suh, 2015). The ease of screening large mutant collections enabled identification of the majority of eceriferum mutants and their characterization. Advancements in targeted mutagenesis, predominantly the CRISPR-Cas technology, opened the way for exploiting other species such as tree tobacco in cuticular lipids research. Apart from outstanding advantages including an extremely high wax load, simplicity of wax composition and induced wax accumulation following drought, tree tobacco is readily transformed, can grow in a variety of conditions including natural settings, and recently had its genome sequenced (Usadel et al., 2018). Furthermore, being a perennial plant, tree tobacco can be explored at both the juvenility stage as well as upon maturation and bark formation. Hence, several key results reported here could not have been discovered in Arabidopsis, such as stem cracking and drought experiments in which plants accumulated tens of grams of biomass and transpired hundreds of milliliters each day.

\section{Alkanes reduce cuticular water loss, but cutin affects photosynthetic sufficiency and light response}

In this study, we simultaneously characterized a large number of mutants corresponding to different cuticular lipids genes. By doing so, we filtered out indirect effects not related to epicuticular wax composition such as changes in cutin composition. Both cerl and the kcs6 mutants possess an extremely reduced alkane load, yet, cerl also retains an altered cutin composition, with significantly less phenols and more $\omega$-hydroxylated fatty acids. Comparing the phenotype of cerl to that of kcs6 enabled us to make the distinction. Cuticular water loss is affected when both genes are mutated, although the rate in cerl is higher. Here we detected an additive effect of altered cutin despite the major effect being that of alkane abundance. Effects that are only present in cer 1 and not kcs6 and which we attribute to cutin rather than epicuticular wax are the reduced $\mathrm{Vc}_{\max }$ and lower carbon assimilation with rising light intensity. In contrast to these, drought response in cerl and kcs6 plants was nearly identical, suggesting that alkane abundance is a major factor essential for recovery following drought. 
It was previously shown that overall accumulation of epicuticular waxes following exposure to drought in tree tobacco reduced the rate of cuticular water loss (Cameron et al., 2006). However, Cameron et al. could not determine which of the many wax constituents underlays this reduction in water loss rate. Here we point to alkanes being the component preventing cuticular water loss. While the correlation of alkanes with water loss rate is high, it does not seem to be linear. The cer3 mutants possess $\sim 20 \%$ of the WT $\mathrm{C}_{31}$ alkane and had only a slight increase in water loss rate. This discrepancy could be observed when removing the cer3 lines from the regression, which raises the $\mathrm{R}^{2}$ from 0.79 to an 0.95 value. It therefore seems that although alkanes reduce cuticular water loss and are essential for recovery following drought, the threshold amount of these components required for having an effect may be well under the one present in WT plants.

\section{Alkane abundance is essential for recovery following drought but does not contribute to fitness under well-watered conditions}

Following the results of the cuticular water loss assay performed on detached leaves, we anticipated that assaying whole plants would mirror this at least partially, and that the mutant plants would transpire at a higher rate under well-watered conditions. This is in line with many studies reporting epicuticular wax conferring drought tolerance, achieved in many cases due to reduced transpiration (reviewed in Xue et al., 2017). Our conclusions from three independent whole plant growth and drought experiments do not match the common view point that wax simply confers drought tolerance by reducing transpiration rates. Plants from our mutant collection, regardless of the metabolic impact of the mutation (whether having no fatty alcohols, no alkanes or a reduced alkane load) all transpire and accumulate biomass at a rate similar to WT under well-watered conditions. Furthermore, although cerl responded to drought by reducing stomatal conductance at a lower soil water content (a phenotype associated with reduced drought tolerance; Negin and Moshelion, 2017), such a response was not detected in kcs6 plants. However, this was not the case with the mutants' recovery dynamics, in which extreme differences could be seen between plants, clustering according to their wax composition. We found that the underlying phenotype that determined whether plants would recover transpiration and growth rapidly following irrigation resumption was leaf survival. We observed clearly that plants that did not recover, were lines in which the drought caused leaves to dry out and die (Fig. S13). Far mutant plants in contrast to alkane 
reduced ones, recovered at the fastest rate, and lost almost no leaves during the drought treatment.

In our drought assay, soil water content and transpiration were continually monitored and plants were resuscitated once they had reached the same transpirational threshold. In contrast, many studies assay drought by stopping irrigation for a similar duration of time (see for example Bourdenx et al., 2011). This experimental design causes plants that have higher transpiration under well-watered conditions to reach drought conditions at an earlier time, and be exposed to drought conditions for a longer period than their counterparts. Furthermore, "drought tolerance" attributed to altered drought response may in fact be secondary to attenuation of other stress conditions. These stress conditions may include high light intensity, temperature and VPD. For example, plants that are able to disperse light rather than absorb it may have a lower leaf temperature, which may be compensated for by increasing transpiration (Richards et al., 1986), making these plants less tolerant to drought induced by a similar duration of irrigation stoppage. Our experimental setup uncoupled wellwatered transpiration and growth from drought response and recovery. This enabled us to point to the ability to strongly reduce transpiration under drought and prevent leaf death as alkane dependent and essential for recovery.

\section{Changes in wax composition and the stem cracking phenomena}

The phenomena of stem cracking is known in conifers at the end of growing seasons of drought years, and increases with drought severity (Zeltińš et al., 2018; Cameron, 2019). However, to the best of our knowledge it has never been linked to alterations in epicuticular wax abundance or composition. Its appearance in kcs6 and cerl mutants following recovery indicates that stem cracking is indeed alkane related. The extensive use of annual plants, mainly Arabidopsis for molecular research of epicuticular waxes, has left its effect on perennial and woody plants relatively unexplored. Apart from presenting an intriguing phenotype linked to deficiencies in epicuticular wax biosynthesis, our study here underscores the use of $N$. Glauca as a model system enabling research of wax function in woody species. The exact stage at which stem cracking took place is not clear to us. Even at the end of the drought treatment, we could not detect cracked stems in the kcs6 plants. However, whether small initial cracking already began during drought, if a more gradual recovery would have prevented the cracking, and what is the threshold of drought conditions resulting in cracking, remains to be determined. 


\section{Glossy mutants did not share a common phenotype under the examined conditions}

Out of the five mutants examined in this study, four display a glossy phenotype. This striking phenotype appears in plants that lost alkane biosynthesis nearly entirely but also when the $\mathrm{C}_{31}$ alkane is greatly reduced in cer3 mutants, and even in the far mutants that are deficient merely in primary alcohols. The far mutants do not exhibit an elevated rate of cuticular water loss. More strikingly, their recovery following drought is greatly improved compared to WT indicating that fatty alcohols are completely unnecessary for drought response. This leads to the question of what is the disadvantage in losing primary alcohols specifically and exhibiting a glossy appearance? Since the glossy phenotype is dependent on optical parameters, we examined photosynthetic efficiency as well as light response. In both cases, there was no correlation between leaf glossiness and these parameters. In eucalyptus species, it was shown that glaucous leaves had the effect of reducing photosynthesis prior to saturating conditions. In addition, in these plants, once wax was removed, photoinhibition occurred at high light intensity while this did not occur in normal plants (Cameron, 1970). Although our initial hypothesis was similar to the findings of Cameron both in terms of reaching photoinhibition at a lower light intensity as well as having higher carbon assimilation at a non-saturating light intensity, this was not the case in the four mutants examined in this study. The cerl mutant line even assimilated carbon at lower rates with rising light intensity.

Epicuticular wax has been suggested to play a protective role against a range of stresses. Drought (Aharoni et al., 2004; Seo et al., 2011; Lee et al., 2014; Lee and Suh, 2015; Xue et al., 2017), UV radiation (Long et al., 2003), osmotic stress (Liu et al., 2019) and insect herbivory (reviewed in Eigenbrode and Espelie, 1995) are a few such conditions. In addition, since epicuticular wax repels water it has been suggested to have an anti-adhesive selfcleaning affect (also known as the "lotus effect"; Neinhuis and Barthlott, 1997), which causes different particles including pathogens to be washed away upon leaf wetting. However, under our experimental conditions, we did not find a common disadvantage in coping with abiotic stress conditions which is linked to a glossy appearance. Hence, we suggest that a glaucous appearance is likely to bear positive effect in combating biotic stress conditions rather than under abiotic ones.

\section{Conclusions}


The examination of epicuticular wax mutants in $N$. glauca revealed that while alkane accumulation is strongly correlated with cuticular water loss, under well-watered conditions epicuticular wax composition does not affect whole plant water loss or plant growth rate. Despite this, the presence of alkanes greatly affected the ability of plants to recover following drought conditions, and its deficiency led to a previously unobserved phenotype of stem cracking. Furthermore, under our experimental conditions, we could not find a common denominator between plants possessing a glossy phenotype suggesting that a glaucous appearance is associated with combating biotic stress conditions. In contrast to this, plants in which cutin was strongly affected were the only ones whose photosynthetic efficiency and light response were altered, emphasizing the importance of uncoupling cutin related affects from those which are epicuticular wax derived.

\section{Materials and methods}

\section{Plant material and growth conditions}

Seeds of $N$. glauca were initially collected from plants growing nearby the Weizmann institute campus in Rehovot, Israel (31.912408, 34.820184). Plants used for transcriptome analysis were grown in the greenhouse (winter 2013). Plants used in cuticular wax and cutin extraction, SEM, stomatal analysis, cuticular water loss and photosynthetic efficiency assays were grown in a greenhouse in two batches during the winter-spring of 2019 and of 2020. Plants used for light curves were grown in a growth room under a flux of $\sim 150 \mu \mathrm{E}$, with a temp. of $22^{\circ} \mathrm{C}$ and light/ dark period of $16 / 8 \mathrm{~h}$. Drought experiments were performed in the lysimetric facility in the Faculty of Agriculture in Rehovot, Israel (31.904134, 34.801060), during 7-9.2018, 1-3.2019 and 3-4.2020.

\section{CRISPR vectors and mutation analysis}

Construct assembly was performed using the 'golden braid' cloning system (SarrionPerdigones et al., 2013). A codon optimized Streptococcus pyogenes Cas9 (Fauser et al., 2014) was used, driven by the Solanum lycopersicum UBIQUITIN10 promoter (Dahan-Meir et al., 2018). All gRNA transcription was driven by the Arabidopsis U6-26 promoter. crRNAs were planned based on the genome assembly of Usadel et al. (2018) using the Crispr-p software (Lei et al., 2014). crRNA sequences, gene maps and transformation 
protocol appear in supplementary methods and Fig. S2-S10. Mutations were analyzed by DNA extraction and PCR amplification using oligonucleotides flanking the expected edited region. In plants where the crRNAs were positioned far from each other on the genome, two PCRs with different primer sets were performed and PCR products sequenced. For homozygous mutations, NCBI blast (https://blast.ncbi.nlm.nih.gov/Blast.cgi) was used to compare the mutant's sequence to WT sequence and in heterozygous cases, they were characterized using one of two softwares: DSDecode (Liu et al., 2015) or CRISP-ID (Dehairs et al., 2016).

\section{Transcriptomics and differential gene analysis}

Transcriptome analysis was performed on plants from several tissues under well-watered and drought conditions [induced by three events of drying and recovery as described in Cameron et al. (2006)]. RNA extraction, library preparation and RNA-seq were performed as described in Hen-Avivi et al. (2016). Since at the time of analysis of the raw reads, the $N$. glauca genome was unpublished, we performed de-novo transcriptome assembly based on Haas et al. (2013) of N. glauca gene expression data published by Long et al. (2016). Based on this assembly, genes were aligned and their differential expression analyzed. This data was then used to define conditions of epidermis enrichment and drought induction, and contigs in which at least three conditions (adaxial epidermis enrichment, abaxial epidermis enrichment and adaxial epidermis drought induction for example) were searched using BlastX against the NCBI protein sequence database (NR) to find their closest homologs.

\section{Wax and cutin monomer profiling}

For wax extraction, three leaf discs with a diameter of $12 \mathrm{~mm}$ each were dipped in $4 \mathrm{ml}$ chloroform with an internal standard of $10 \mu \mathrm{g} \mathrm{C} 36$ alkane and shaken gently for $15 \mathrm{sec}$. The discs were then removed and chloroform was evaporated under a nitrogen flow. Samples were then resuspended in $100 \mu \mathrm{l}$ chloroform to which $20 \mu \mathrm{L}$ pyridine and $20 \mu \mathrm{L}$ BSTFA were added. Samples were derivatized at $70^{\circ} \mathrm{C}$ and injected in a splitless mode to a GC-MS system (Agilent 7890A chromatograph, 5975C mass spectrometer and a 7683 auto sampler) as described in Cohen et al. (2019). Chromatograms and mass spectra were analyzed using MSD Chemstation software (Agilent). Identification was based both on fragmentation alignment to the NIST Mass Spectral Library and in-house retention Indexes. Quantification was performed using the Chemstation software and was normalized to the $\mathrm{C}_{36}$ alkane internal 
standard. Cutin extraction was performed as described in Cohen et al. (2019). Monomer identification and quantification was performed as in wax components analysis, with normalization performed against a $\mathrm{C}_{32}$ alkane internal standard.

\section{Leaf desiccation assays and whole plant drought trials}

Five leaves from different plants from each independent line were cut in the greenhouse and immediately inserted to zip-locked bags, brought to the lab and let dry at $22^{\circ} \mathrm{C}$. Leaves were weighed every two hours for 12 hours. This was done following an earlier calibration in which leaves were weighed every half hour and which showed that following two hours of desiccation water loss was linear up to $24 \mathrm{~h}$. Due to the large number of leaves and need to repeatedly weigh them at given intervals, the experiment was divided to three different days, each having its own WT control. Drought trials performed using a lysimetric system (during the summer of 2018, winter of 2019 and spring of 2020) were performed in either soil (2018, 2019), or sand (2020) as described in Halperin et al. (2016) and Dalal et al. (2020) (see supplementary methods for details).

\section{Stomatal analysis and scanning electron microscopy}

Stomatal imprints were taken from adaxial and abaxial epidermises of greenhouse grown plants, as described in Yaaran et al. (2019). Nail polish imprints of dental resin were then attached to microscope slides and photographed in a Nikon eclipse E800 microscope with a Nikon Digital sight DS-5Mc camera. Each imprint was photographed at two different locations at a magnification of $\mathrm{x} 100$ for stomatal density and at another two at a magnification of $\mathrm{x} 400$ for stomatal aperture and size. Stomata were then quantified and aperture and size were measured using ImageJ software (https://imagej.nih.gov/ij/). For SEM, small sections were cut from fresh leaves of WT and representative lines from the five mutant genes and inserted to a cryo-holder, frozen in liquid nitrogen, coated and photographed using a Zeiss Ultra 55 SEM as described in Hen-Avivi et al. (2016).

\section{Photosynthetic efficiency and light response}

Photosynthetic efficiency was assessed by measuring gas exchange at rising $\mathrm{CO}_{2}$ concentrations ("A/Ci curves"), using a Li-6800 portable photosynthesis system (LI-COR, Inc.; Lincoln, NE, USA). Parameters were set to: PAR 1,600, relative humidity $60 \%, \mathrm{CO}_{2}$ 400PPM and leaves were inserted to the infrared gas analyzer (IRGA) chamber for 
acclimation. This stage was performed for at least $15 \mathrm{~min}$ and was stopped when carbon assimilation ceased rising exponentially (but no longer than $25 \mathrm{~min}$ ). A/Ci curves were then performed with the points: 400PPM, 300PPM, 150PPM, 50PPM, 0, 400PPM, 600PPM, 800PPM, 1,000PPM, 1,200PPM and 1,500PPM CO 2 . Data was analyzed using R (www.rproject.org) and the 'plantecophys' package (Duursma, 2015). Light response was measured using the Li-6800 portable photosynthesis system. Parameters were similar to those used for A/Ci curves, excluding light intensity which was raised every 6 min. Plants were first dark acclimated by covering the leaf on which the measurement would be performed in aluminum foil for 10min, after which the leaf was inserted to the IRGA chamber. Each light intensity was kept for at least $6 \mathrm{~min}$, and once $\mathrm{A}$ and $\mathrm{g}_{\mathrm{s}}$ were stable a measurement was taken. The light increment program was as follows: $0,10 \mu \mathrm{E}, 20 \mu \mathrm{E}, 50 \mu \mathrm{E}, 100 \mu \mathrm{E}, 200 \mu \mathrm{E}, 400 \mu \mathrm{E}, 800$ $\mu \mathrm{E}, 1,200 \mu \mathrm{E}, 1,600 \mu \mathrm{E}, 2,000 \mu \mathrm{E}$ and 2,200 $\mu \mathrm{E}$.

\section{Statistical analysis}

The JMP 14 software (SAS Institute; http://www.jmp.com/en_us/home.html) was used for all statistical analyses, except for two-piece linear curves in the drought experiments in which in-house statistical tools of the lysimeter system (https://www.plant-ditech.com/) were used to find the best fitting regression lines. Student's $t$ test was used when comparing two groups. Mutants were always compared to WT, except in the spring drought trial where Tukey HSD test was used and all groups were compared.

\section{Acknowledgments}

We thank Prof. Björn Usadel for providing us with access to unpublished $N$. glauca genomic and transcriptomic data. These high quality assemblies aided us to a great extent throughout this study. 


\section{References}

Aarts MG, Keijzer CJ, Stiekema WJ, Pereira A (1995) Molecular characterization of the CERl gene of Arabidopsis involved in epicuticular wax biosynthesis and pollen fertility. Plant Cell. 7: 2115-2127

Aharoni A, Dixit S, Jetter R, Thoenes E, van Arkel G, Pereira A (2004) The SHINE clade of AP2 domain transcription factors activates wax biosynthesis, alters cuticle properties, and confers drought tolerance when overexpressed in Arabidopsis. Plant Cell 16: 24632480

Bach L, Michaelson L V., Haslam R, Bellec Y, Gissot L, Marion J, Da Costa M, Boutin JP, Miquel M, Tellier F, et al (2008) The very-long-chain hydroxy fatty acyl-CoA dehydratase PASTICCINO2 is essential and limiting for plant development. Proc Natl Acad Sci U S A 105: 14727-14731

\section{Barthlott W, Neinhuis C, Cutler D, Ditsch F, Meusel I, Theisen I, Wilhelmi H (1998)} Classification and terminology of plant epicuticular waxes. Bot J Linn Soc. 126: 237260

\section{Beaudoin F, Wu X, Li F, Haslam RP, Markham JE, Zheng H, Napier JA, Kunst L} (2009) Functional characterization of the Arabidopsis $\beta$-ketoacyl-coenzyme a reductase candidates of the fatty acid elongase. Plant Physiol 150: 1174-1191

Bickford CP (2016) Ecophysiology of leaf trichomes. Funct Plant Biol 43: 807-814

Bourdenx B, Bernard A, Domergue F, Pascal S, Leger A, Roby D, Pervent M, Vile D, Haslam RP, Napier JA, et al (2011) Overexpression of Arabidopsis ECERIFERUM1 promotes wax very-long-chain alkane biosynthesis and influences plant response to biotic and abiotic stresses. Plant Physiol 156: 29-45

Cameron AD (2019) Mitigating the risk of drought-induced stem cracks in conifers in a changing climate. Scand J For Res 34: 667-672

Cameron KD, Teece MA, Smart LB (2006) Increased accumulation of cuticular wax and expression of lipid transfer protein in response to periodic drying events in leaves of tree tobacco. Plant Physiol 140: 176-183

Cameron RJ (1970) Light intensity and the growth of Eucalyptus seedlings. II. The effect of cuticular waxes on light absorption in leaves of Eucalyptus species. Aust J Bot 18: 275284

Chalker-Scott L (1999) Environmental significance of anthocyanins in plant stress 
responses. Photochem Photobiol 70: 1-9

Chen X, Goodwin SM, Boroff VL, Liu X, Jenks MA (2003) Cloning and characterization of the WAX2 gene of Arabidopsis involved in cuticle membrane and wax production. Plant Cell 15: 1170-1185

Cohen H, Dong Y, Szymanski J, Lashbrooke J, Meir S, Almekias-Siegl E, Zeisler-Diehl VV, Schreiber L, Aharoni A (2019) A multilevel study of melon fruit reticulation provides insight into skin ligno-suberization hallmarks. Plant Physiol 179: 1486-1501

Dahan-Meir T, Filler-Hayut S, Melamed-Bessudo C, Bocobza S, Czosnek H, Aharoni A, Levy AA (2018) Efficient in planta gene targeting in tomato using geminiviral replicons and the CRISPR/Cas9 system. Plant J 95: 5-16

Dalal A, Shenhar I, Bourstein R, Mayo A, Grunwald Y, Averbuch N, Attia Z, Wallach R, Moshelion M (2020) A high-throughput gravimetric phenotyping platform for realtime physiological screening of plant-environment dynamic responses. bioRxiv. doi: 10.1101/2020.01.30.927517

Dehairs J, Talebi A, Cherifi Y, Swinnen J V. (2016) CRISP-ID: Decoding CRISPR mediated indels by Sanger sequencing. Sci Rep 6: 1-5

Duursma RA (2015) Plantecophys - An R package for analysing and modelling leaf gas exchange data. PLoS One 10: e0143346

Eigenbrode SD, Espelie KE (1995) Effects of plant epicuticular lipids on insect herbivores. Annu Rev Entomol 40: 171-194

Fauser F, Schiml S, Puchta H (2014) Both CRISPR/Cas-based nucleases and nickases can be used efficiently for genome engineering in Arabidopsis thaliana. Plant J 79: 348-359

Greer S, Wen M, Bird D, Wu X, Samuels L, Kunst L, Jetter R (2007) The cytochrome P450 enzyme CYP96A15 is the midchain alkane hydroxylase responsible for formation of secondary alcohols and ketones in stem cuticular wax of Arabidopsis. Plant Physiol 145: $653-667$

Haas BJ, Papanicolaou A, Yassour M, Grabherr M, Blood PD, Bowden J, Couger MB, Eccles D, Li B, Lieber M, et al (2013) De novo transcript sequence reconstruction from RNA-seq using the Trinity platform for reference generation and analysis. Nat Protoc $\mathbf{8}$ : 1494-1512

Halperin O, Gebremedhin A, Wallach R, Moshelion M (2016) High-throughput physiological phenotyping and screening system for the characterization of plantenvironment interactions. Plant J. 89: 839-850

Hanley ME, Lamont BB, Fairbanks MM, Rafferty CM (2007) Plant structural traits and 
their role in anti-herbivore defence. Perspect Plant Ecol Evol Syst 8: 157-178

Hen-Avivi S, Savin O, Racovita RC, Lee W-S, Adamski NM, Malitsky S, Almekias-Siegl

E, Levy M, Vautrin S, Bergès H, et al (2016) A Metabolic Gene Cluster in the Wheat

W1 and the Barley Cer-cqu Loci Determines b-Diketone Biosynthesis and

Glaucousness. Plant Cell 28: 1440-1460

Jetter R, Riederer M (2016) Localization of the transpiration barrier in the epi- and intracuticular waxes of eight plant species: Water transport resistances are associated with fatty acyl rather than alicyclic components. Plant Physiol 170: 921-934

Lee SB, Kim H, Kim RJ, Suh MC (2014) Overexpression of Arabidopsis MYB96 confers drought resistance in Camelina sativa via cuticular wax accumulation. Plant Cell Rep 33: $1535-1546$

Lee SB, Suh MC (2015) Advances in the understanding of cuticular waxes in Arabidopsis thaliana and crop species. Plant Cell Rep 34: 557-572

Lei Y, Lu L, Liu HY, Li S, Xing F, Chen LL (2014) CRISPR-P: A web tool for synthetic single-guide RNA design of CRISPR-system in plants. Mol Plant 7: 1494-1496

Li F, Wu X, Lam P, Bird D, Zheng H, Samuels L, Jetter R, Kunst L (2008) Identification of the wax ester synthase/acyl-coenzyme a:diacylglycerol acyltransferase WSD1 required for stem wax ester biosynthesis in Arabidopsis. Plant Physiol 148: 97-107

Liu N, Chen J, Wang T, Li Q, Cui P, Jia C, Hong Y (2019) Overexpression of WAX INDUCER1/SHINE1 gene enhances wax accumulation under osmotic stress and oil synthesis in Brassica napus. Int J Mol Sci. 20: 4435

Liu W, Xie X, Ma X, Li J, Chen J, Liu YG (2015) DSDecode: A web-based tool for decoding of sequencing chromatograms for genotyping of targeted mutations. Mol Plant 8: $1431-1433$

Long LM, Prinal Patel H, Cory WC, Stapleton AE (2003) The maize epicuticular wax layer provides UV protection. Funct Plant Biol 30: 75-81

Long N, Ren X, Xiang Z, Wan W, Dong Y (2016) Sequencing and characterization of leaf transcriptomes of six diploid Nicotiana species. J Biol Res 23: 6

Lü S, Song T, Kosma DK, Parsons EP, Rowland O, Jenks MA (2009) Arabidopsis CER8 encodes LONG-CHAIN ACYL-COA SYNTHETASE 1 (LACS1) that has overlapping functions with LACS2 in plant wax and cutin synthesis. Plant J 59: 553-564

Millar AA, Clemens S, Zachgo S, Giblin EM, Taylor DC, Kunst L, Michael Giblin E, Taylor DC, Kunst L (1999) CUT1, an Arabidopsis gene required for cuticular wax biosynthesis and pollen fertility, encodes a very-long-chain fatty acid condensing 
enzyme. Plant Cell. 5: 825-838

Millar AA, Kunst L (1997) Very-long-chain fatty acid biosynthesis is controlled through the expression and specificity of the condensing enzyme. Plant J 12: 121-131

Mortimer CL, Bramley PM, Fraser PD (2012) The identification and rapid extraction of hydrocarbons from Nicotiana glauca: A potential advanced renewable biofuel source. Phytochem Lett 5: 455-458

Negin B, Moshelion M (2017) The advantages of functional phenotyping in pre-field screening for drought-tolerant crops. Funct Plant Biol 44: 107-118

Neinhuis C, Barthlott W (1997) Characterization and distribution of water-repellent, selfcleaning plant surfaces. Ann Bot 79: 667-677

Richards R., Rawson H., Johnson D. (1986) Glaucousness in wheat: Its development and effect on water-use efficiency, gas exchange and photosynthetic tissue temperatures. Funct Plant Biol 13: 465

Rowland O, Zheng H, Hepworth SR, Lam P, Jetter R, Kunst L (2006) CER4 encodes an alcohol-forming fatty acyl-coenzyme A reductase involved in cuticular wax production in Arabidopsis. Plant Physiol 142: 866-77

Ryan KG, Swinny EE, Markham KR, Winefield C (2002) Flavonoid gene expression and UV photoprotection in transgenic and mutant Petunia leaves. Phytochemistry. 59: 23-32 Ryan KG, Swinny EE, Winefield C, Markham KR (2001) Flavonoids and UV photoprotection in Arabidopsis mutants. Zeitschrift fur Naturforsch - Sect C J Biosci 56: $745-754$

Sarrion-Perdigones A, Vazquez-Vilar M, Palací J, Castelijns B, Forment J, Ziarsolo P, Blanca J, Granell A, Orzaez D (2013) Goldenbraid 2.0: A comprehensive DNA assembly framework for plant synthetic biology. Plant Physiol 162: 1618-1631

Schnurr J, Shockey J, Browse J (2004) The Acyl-CoA synthetase encoded by LACS2 Is essential for normal cuticle development in Arabidopsis. Plant Cell 16: 629-642

Seo PJ, Lee SB, Suh MC, Park MJ, Park CM (2011) The MYB96 transcription factor regulates cuticular wax biosynthesis under drought conditions in Arabidopsis. Plant Cell 23: $1138-1152$

Usadel B, Tohge T, Scossa F, Sierro N, Schmidt M, Vogel A, Bolger A, Kozlo A, Enfissi EM, Morrel K, et al (2018) The genome and metabolome of the tobacco tree, Nicotiana glauca : a potential renewable feedstock for the bioeconomy. bioRxiv 351429 Xue D, Zhang X, Lu X, Chen G, Chen ZH (2017) Molecular and evolutionary mechanisms of cuticular wax for plant drought tolerance. Front Plant Sci. 8: 621 
Yaaran A, Negin B, Moshelion M (2019) Role of guard-cell ABA in determining steadystate stomatal aperture and prompt vapor-pressure-deficit response. Plant Sci 281: 31-40

Yeats TH, Rose JKC (2013) The formation and function of plant cuticles. Plant Physiol 163: $5-20$

Zeisler-Diehl V, Müller Y, Schreiber L (2018) Epicuticular wax on leaf cuticles does not establish the transpiration barrier, which is essentially formed by intracuticular wax. $\mathbf{J}$ Plant Physiol 227: 66-74

Zeisler V, Schreiber L (2016) Epicuticular wax on cherry laurel (Prunus laurocerasus) leaves does not constitute the cuticular transpiration barrier. Planta 243: 65-81

Zeltińš P, Katrevičs J, Gailis A, Maaten T, Baders E, Jansons A (2018) Effect of stem diameter, genetics, and wood properties on stem cracking in Norway spruce. Forests 9: 546

Zheng H, Rowland O, Kunst L (2005) Disruptions of the Arabidopsis enoyl-CoA reductase gene reveal an essential role for very-long-chain fatty acid synthesis in cell expansion during plant morphogenesis. Plant Cell 17: 1467-1481 